\title{
Lifted Heegaard Surfaces and Virtually Haken Manifolds
}

\author{
Yu Zhang* \\ University at Buffalo, The State University of New York
}

\begin{abstract}
In this paper, we give infinitely many non-Haken hyperbolic genus three 3-manifolds each of which has a finite cover whose induced Heegaard surface from some genus three Heegaard surface of the base manifold is reducible but can be compressed into an incompressible surface. This result supplements [CG] and extends [MMZ.
\end{abstract}

\section{Introduction}

It was shown in $\mathrm{CG}$ that if a Heegaard splitting of an irreducible closed 3-manifold $M$ is weakly reducible then either the Heegaard splitting is reducible or $M$ contains an incompressible surface of positive genus. This result motivates an approach to the well known virtual Haken conjecture which, with the current knowledge, is reduced to the following conjecture: every closed hyperbolic 3-manifold is virtually Haken, i.e. has a finite cover which is a Haken 3-manifold. That is, to prove that a given closed hyperbolic 3-manifold is virtually Haken, it suffices to find a finite cover which has an irreducible but weakly reducible Heegaard splitting. In [MMZ], families of non-Haken but virtually Haken hyperbolic 3-manifolds were found using this approach. These manifolds were obtained by Dehn surgeries on some 2-bridge knots in $S^{3}$ and thus are genus two 3-manifolds. In fact it was showed there that each of these manifolds has a finite cover whose induced Heegaard surface from some genus two Heegaard surface of the base manifold is weakly reducible and can be compressed into an incompressible surface, without the need to know whether the Heegaard surface of the cover is irreducible or not (we suspect that it is irreducible).

The main purpose of this paper is to illustrate two points concerning the above works. One point is to show that the method used in [MMZ can be generalized to find an infinite family of closed non-Haken but virtually Haken hyperbolic genus three 3-manifolds. The other point is to show that each manifold of our family has a finite cover whose induced Heegaard surface from some genus three Heegaard surface of the base manifold is actually reducible but can still be compressed into an incompressible surface, which is a phenomenon

*E-mail: yz26@buffalo.edu 
supplementing [CG]. Our manifolds are obtained by Dehn surgeries on some pretzel knots in $S^{3}$.

Let $K=(p, \pm 3, q)$ be a pretzel knot in $S^{3}$ with $p, q$ odd and $|p|,|q| \geqslant 3$, and let $M_{K}=S^{3} \backslash \stackrel{\circ}{N}(K)$ be the exterior of $K$. Let $M_{K}^{3}$ be the 3 -fold cyclic cover of $M_{K}$. We give $\partial M_{K}$ the standard meridian-longitude coordinates and $\partial M_{K}^{3}$ the induced meridianlongitude coordinates. So a slope in such a torus can be identified with a rational number $m / n$ where $m$ is the meridian coordinate and $n$ the longitude coordinate. By [La], $K$ is a tunnel number two knot and thus $M_{K}$ is a genus three manifold.

Theorem 1.1. For the pretzel knot $K=(p, \pm 3, q)$, the induced Heegaard surface of $M_{K}^{3}$ from some genus three Heegaard surface of $M_{K}$ is reducible and can be compressed into an essential surface $S$ in $M_{K}^{3}$. Moreover, $S$ remains essential in every Dehn filling of $M_{K}^{3}$ with slope $m / n,(m, n)=1,|m| \geqslant 2$. Thus every Dehn filling of $M_{K}$ with slope $3 m / n,(3 m, n)=$ $1,|m| \geqslant 2$, yields a virtually Haken 3 -manifold.

We now explain how the results described in the second paragraph of this section follow from Theorem 1.1. As we have noted, $M_{K}$ is a genus three manifold. Thus every Dehn filling of $M_{K}$ is of genus at most three. As $K$ is a hyperbolic small knot by [0] (here small means no closed embedded essential surfaces in $M_{K}$ ), it follows from [RS] that except for finitely many lines in the Dehn filling plane of $M_{K}$, all remaining Dehn fillings of $M_{K}$ are genus three manifolds, which we may also assume to be

(1) hyperbolic, by Thurston's hyperbolic Dehn surgery theorem, and

(2) non-Haken, by [H].

Hence infinitely many of $M_{K}(3 \mathrm{~m} / n)$ given in Theorem 1.1 are genus three non-Haken hyperbolic 3-manifolds. Finally we just need to note that $M_{K}(3 m / n)$ is covered by $M_{K}^{3}(m / n)$ and that each Heegaard splitting of $M_{K}$ induces a Heegaard splitting on $M_{K}(3 \mathrm{~m} / n)$.

The proof of Theorem 1.1 is given in Section 3, after some preliminary preparations in Section 2. Using a similar method, we shall also give a new proof of [O, Corollary 4(b)] in case of pretzel knots. This is the content of Section 4 .

\section{Preliminary}

Heegaard Splittings. A Heegaard splitting $M=W_{1} \cup_{F} W_{2}$ of a compact 3-manifold $M$ is a decomposition of $M$ into two compression bodies $W_{1}$ and $W_{2}$ with common positive boundary $F$. A Heegaard splitting $M=W_{1} \cup_{F} W_{2}$ is reducible if there exist essential disks $\left(D_{1}, \partial D_{1}\right) \subset\left(W_{1}, F\right)$ and $\left(D_{2}, \partial D_{2}\right) \subset\left(W_{2}, F\right)$ such that $\partial D_{1}=\partial D_{2}$. Otherwise, it is irreducible. If neither $W_{1}$ nor $W_{2}$ is trivial and there do not exist essential disks $\left(D_{1}, \partial D_{1}\right) \subset\left(W_{1}, F\right)$ and $\left(D_{2}, \partial D_{2}\right) \subset\left(W_{2}, F\right)$ such that $\partial D_{1} \cap \partial D_{2}=\emptyset$, then the Heegaard splitting is strongly irreducible. Otherwise, it is weakly reducible. We call a Heegaard splitting $M=W_{1} \cup_{F} W_{2}$ stabilized if there exist essential disks $\left(D_{1}, \partial D_{1}\right) \subset\left(W_{1}, F\right)$ and 


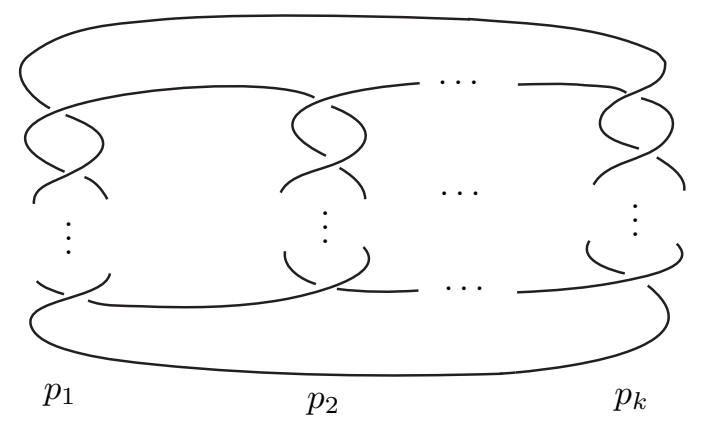

Figure 1: Pretzel link

$\left(D_{2}, \partial D_{2}\right) \subset\left(W_{2}, F\right)$ such that $\partial D_{1}$ and $\partial D_{2}$ intersect at a single point. It is known that every reducible splitting of an irreducible manifold is stabilized.

Pretzel Links. A pretzel link is a special kind of link. A pretzel link which is also a knot is a pretzel knot. In the standard projection of the $\left(p_{1}, p_{2}, \cdots, p_{k}\right)$-pretzel link, there are $p_{i}$ left-handed crossings in the $i$ th tangle, see Figure 1. Obviously, the $\left(p_{1}, p_{2}, \cdots, p_{k}\right)$-pretzel link is link-equivalent to the $\left(p_{i}, p_{i+1}, \cdots, p_{k}, p_{1}, \cdots, p_{i-1}\right)$-pretzel link.

For a pretzel link $K=\left(p_{1}, p_{2}, \cdots, p_{k}\right)$, the number of components $|K|$ of $K$ is given by

$$
|K|= \begin{cases}1 & \text { if each } p_{i} \text { is odd and } k \text { is odd } \\ 2 & \text { if each } p_{i} \text { is odd and } k \text { is even } \\ \#\left\{i: p_{i} \text { is even }\right\} & \text { if some } p_{i} \text { 's are even. }\end{cases}
$$

The Whitehead Graphs. Let $H$ be a handlebody. A finite set of pairwise disjoint simple closed curves $A$ in $\partial H$ is said to be separable in $H$ if and only if $\partial H-A$ is compressible in $H$. Let $D$ be a compression disk system of $H$, i.e., a set of embedded essential disks in $H$ which compress $H$ into a 3-ball $B$. Each disk $d$ in $D$ has two copies $d^{+}, d^{-}$in $\partial B$. The Whitehead graph of $A \subset \partial H$ with respect to $D$, denoted by $W G(D, A)$, is the graph on $\partial B$ taking $\cup d^{ \pm}$as vertices and taking the line segments $\left[\partial B-\operatorname{int}\left(\cup d^{ \pm}\right)\right] \cap A$ as edges. It is shown in [S] that if the Whitehead graph is connected and has no cut vertex, then $A$ is non-separable in $H$, and if the graph is disconnected, then $A$ must be separable. When the graph has a cut vertex $v$, a Whitehead automorphism corresponding to $v$ can be made to transform the graph into an equivalent graph (by changing the disk system $D$ ) which has less complexity (i.e. the number of edges). So after a finitely many Whitehead automorphisms, we may end up with a disconnected graph or a connected graph with no cut vertices. We refer to $\underline{\underline{S}}$ for details about how to make the Whitehead automorphism at a cut vertex. The following two elementary lemma and corollary will be handy in the proof of Theorem 1.1 .

Lemma 2.1. If the Whitehead graph of $A \subset \partial H$ consists of as two subgraphs $B$ and $C$ 
(1)

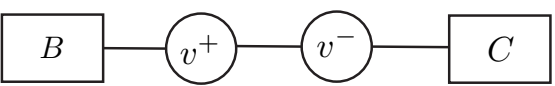

(2)

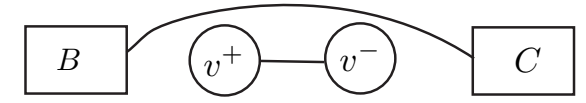

(3)

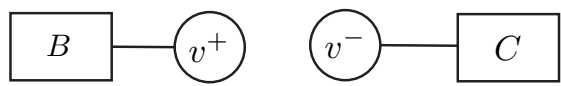

Figure 2: A graph contains a path with $v^{+}$and $v^{-}$.

connected by a path with two vertices $\left\{v^{+}, v^{-}\right\}$(as shown in part (1) of Figure 2), then $A$ is separable in $H$.

Proof: In Figure 2 (1), the vertex $v^{+}$is a cut vertex. Applying the Whitehead automorphism to $v^{+}$, we get a new graph which looks like Figure 2 (2) or (3), both being disconnected. So $A$ is separable.

Corollary 2.2. If the Whitehead graph of $A \subset \partial H$ has a vertex of valence one, then $A$ is separable in $H$.

Lastly in this section we record the Multi-Handle Addition Theorem given in [Le].

Theorem 2.3. [Le] Let $C=\left\{c_{1}, \cdots, c_{n}\right\}$ be a set of pairwise disjoint simple closed curves in the boundary of a handlebody $H$ of genus $k>0$. If the following conditions are satisfied: (0) $\partial H-C$ is incompressible in $H$,

(1) for each $j, \partial H-\left(C-c_{j}\right)$ is compressible in $H$, i.e., $C-c_{j}$ does not bind the free group $F_{k}$,

(p) for any $(n-p)$-element subfamily $C^{\prime}$ of $C, C^{\prime}$ does not bind any free factor $F_{k-p+1}$ of $F_{k}$,

$(n-1)$ for any $c_{j} \in C, c_{j}$ does not bind a free factor $F_{k-n+2}$ of $F_{k}$.

Then the 3-manifold obtained by adding $n$ 2-handles to $H$ along $C$ has incompressible boundary.

See [Le] for the term "bind a free factor".

\section{Proof of Theorem 1.1}

We first give a detailed proof when $K$ is the $(3,3,3)$-pretzel knot (Figure 3 shows its standard diagram) and then indicate how to extend the proof to work for general $K=(p, \pm 3, q)$. 


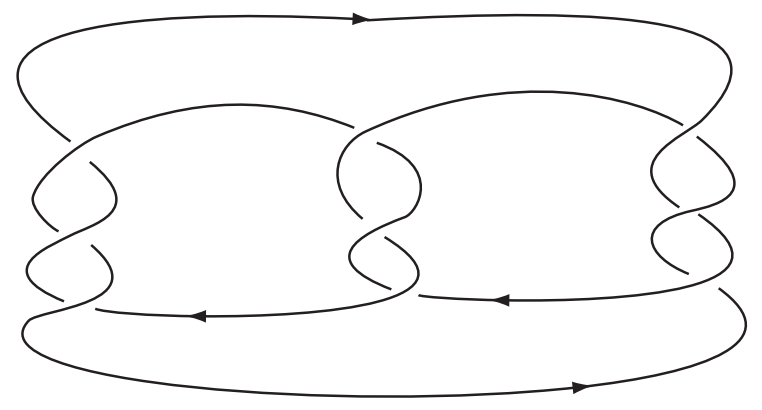

Figure 3: The standard diagram of $(3,3,3)$-pretzel knot

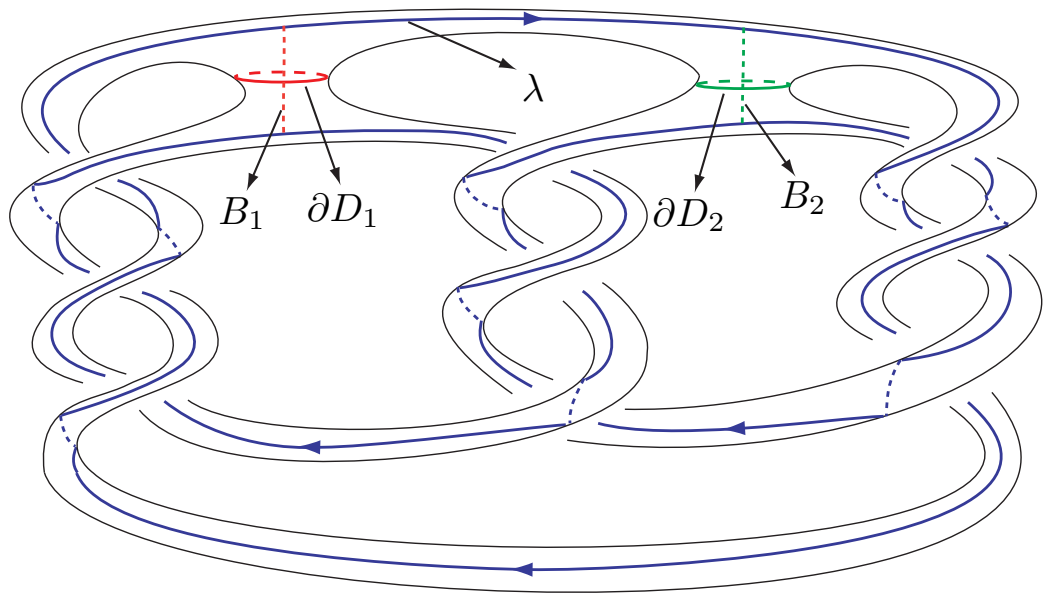

Figure 4: $H$, a regular neighborhood of $K=(3,3,3)$ with unknotting tunnels.

As showed in [La], $K$ is a tunnel number two knot. Figure 4 shows two unknotting tunnels $B_{1}$ and $B_{2}$ for $K$ (noticing here we have more options of the tunnels and we always pick the two as shown in Figure 4), and a regular neighborhood $H$ of $K \cup B_{1} \cup B_{2}$. In the figure, $D_{i}$ is a meridian disk of $N\left(B_{i}\right) . H$ is a handlebody of genus three. We can deform $H$ such that its exterior $H^{\prime}$ is a standard handlebody in $\mathbb{S}^{3}$. At the same time of the deformation, we can keep track of the curves $\partial D_{1}, \partial D_{2}$ and $\lambda$, where $\lambda$ is a standard longitude. Figures 5 5 show the procedure of the deformation: Figure 5 shows the result after we untangle the three crossings on the left, Figure 6 shows the result after we untangle the three crossings on the right, and Figure 7 shows the result after we untangle the three crossings in the middle.

Pick a disk system $\{X, Y, Z\}$ for $H^{\prime}$ such that the boundaries of $X, Y$ and $Z$ are as shown in Figure 7. Let $\{x, y, z\}$ be a generating set for $\pi_{1}\left(H^{\prime}\right)$ dual to disk system, where $x$ is a simple closed curve in $\partial H^{\prime}$ which is disjoint from $\partial Y$ and $\partial Z$ and intersects $\partial X$ exactly 


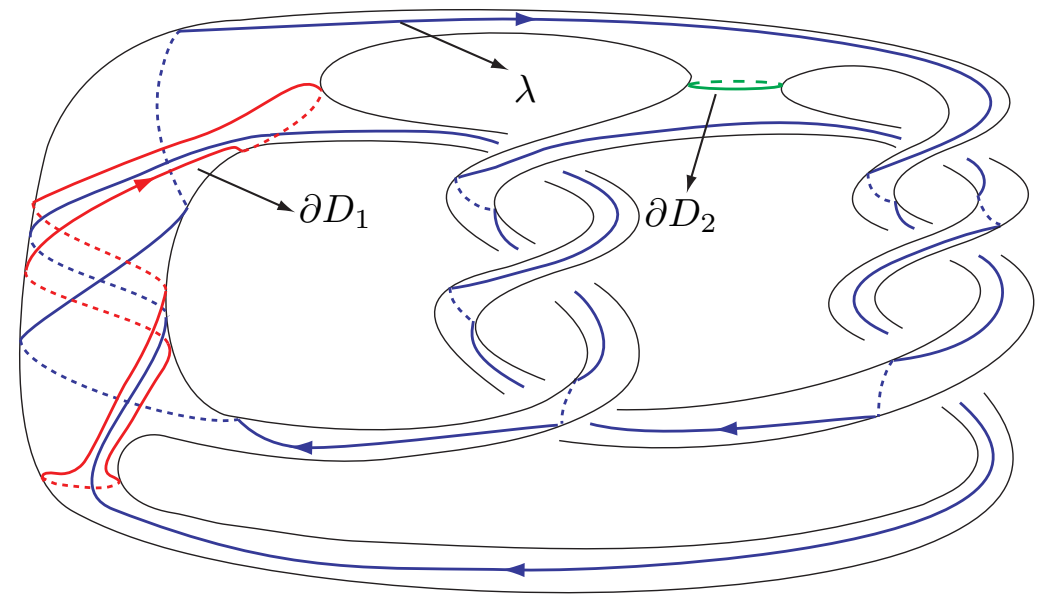

Figure 5: The deformation of $H, \partial D_{1}, \partial D_{2}$ and $\lambda$ (part 1).

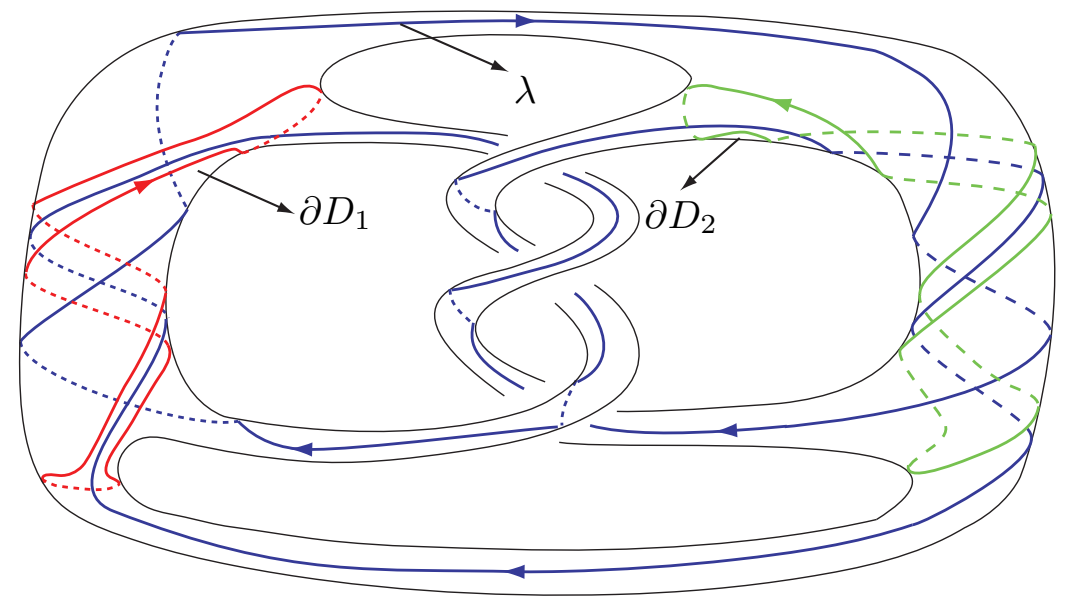

Figure 6: The deformation of $H, \partial D_{1}, \partial D_{2}$ and $\lambda$ (part 2). 


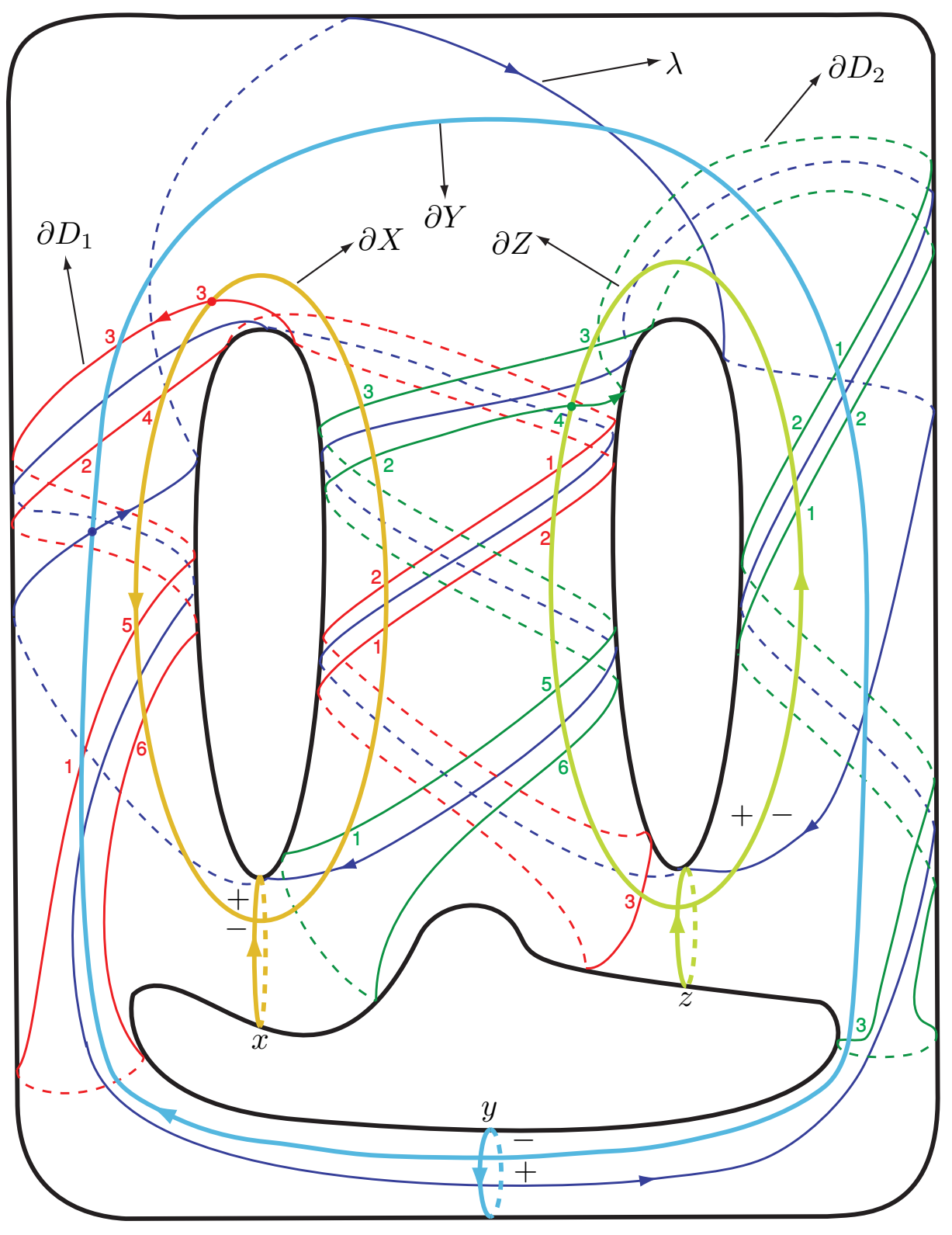

Figure 7: The deformation of $H, \partial D_{1}, \partial D_{2}$ and $\lambda$ (part 3). 
once, and $y$ and $z$ are chosen similarly, as indicated in Figure 7 . Note that in the figure we picked each of $x, y$ and $z$ up to isotopy and didn't draw them as loops sharing a common base point. We orient $x, y$ and $z$ using the right-hand rule with our thumbs pointing to the positive direction of $\lambda$. We also assume that $x$ ( $y, z$ respectively) travels from the negative side to the positive side of $\partial X(\partial Y, \partial Z$ respectively). Then we orient $\partial X, \partial Y$ and $\partial Z$ by the right-hand rule such that our thumbs point to the positive sides. In the figure, we also give indices to the intersection points between $\left\{\partial D_{1}, \partial D_{2}\right\}$ and $\{\partial X, \partial Y, \partial Z\}$ (e.g. $\partial D_{1}$ (in red color) has six intersection points with $\partial X$ and they are labeled by $1,2, . ., 6$ around $\partial X$ (in red colo), other intersection points are labeled in similar way).

Following the given directions, we can write out the expressions of $\partial D_{1}$ and $\partial D_{2}$ in terms of $x, y$ and $z$. They are

$$
\begin{gathered}
\partial D_{1}=\left(x^{-1} y\right)^{2}\left(x y^{-1}\right)\left(x z^{-1}\right)^{2}\left(x^{-1} z\right) \\
\partial D_{2}=\left(z y^{-1}\right)^{2}\left(z^{-1} y\right)\left(z^{-1} x\right)^{2}\left(z x^{-1}\right) .
\end{gathered}
$$

So we get a presentation of the fundamental group $\pi_{1}\left(M_{K}\right)$ of $M_{K}$ :

$$
\pi_{1}\left(M_{K}\right)=\left\langle x, y, z: \partial D_{1}=1, \partial D_{2}=1\right\rangle
$$

By abelinization we get the homology group of $M_{K}$

$$
H_{1}\left(M_{K}\right)=\langle y\rangle
$$

noticing that each of $x$ and $z$ is also a generator of $H_{1}\left(M_{K}\right)$.

By following the given direction, we can also find the expression of the longitude $\lambda$ in terms of the generators $x, y$ and $z$ :

$$
\lambda=\left(y^{-1} x\right)\left(y^{-1} z\right)^{2}\left(x^{-1} z\right)\left(x^{-1} y\right)^{2}\left(z^{-1} y\right)\left(z^{-1} x\right)^{2} .
$$

$M_{K}$ has a Heegaard splitting, $M_{K}=C \cup_{\partial H^{\prime}} H^{\prime}$. Where $C$ is a compression body obtained by attaching two 1-handles $N\left(B_{1}\right)$ and $N\left(B_{2}\right)$ to the positive boundary $\partial M_{K} \times[1]$ of $\partial M_{K} \times[0,1] . \partial H^{\prime}$ is the Heegaard surface (of genus three) and $\left\{\partial D_{1}, \partial D_{2}, \partial X, \partial Y, \partial Z\right\}$ gives us the Heegaard diagram of this splitting, as shown in Figure 7.

Now, let's consider the 3 -fold cyclic cover $M_{K}^{3}$ of $M_{K}$ induced by the homomorphism $h$ from $\pi_{1}\left(M_{K}\right)$ to $\mathbb{Z}_{3}$ factoring through $H_{1}\left(M_{K}\right)$ :

$$
h: \pi_{1}\left(M_{K}\right) \rightarrow \mathbb{Z}_{3} ; x \mapsto \overline{1}, y \mapsto \overline{1}, z \mapsto \overline{1} .
$$

By cutting $\partial H^{\prime}$ open along $\{\partial X, \partial Y, \partial Z\}$ and pasting 3 copies of the resulting surface together cyclicly, we get the induced Heegaard surface of the induced Heegaard splitting of 


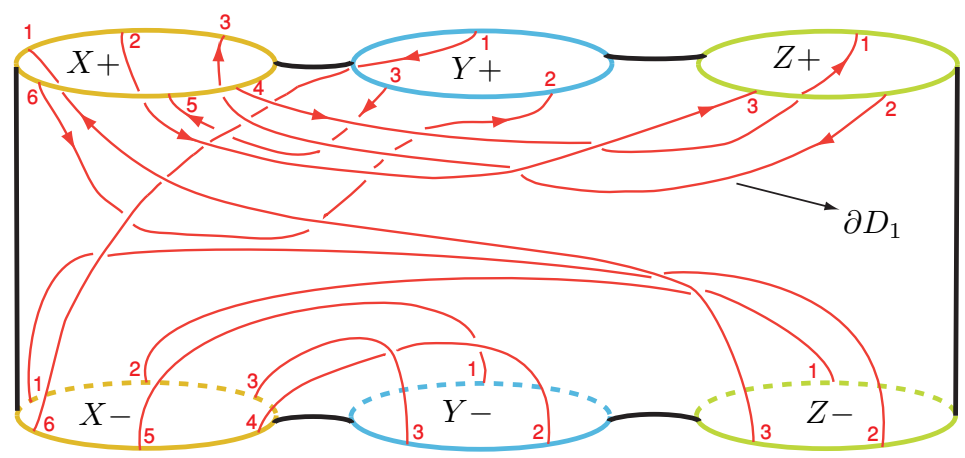

Figure 8: $\partial D_{1}$ in the resulting surface of cutting $\partial H^{\prime}$ open along $\{\partial X, \partial Y, \partial Z\}$.

$M_{K}^{3}$. We show the procedure in Figure 8-Figure 11. Here we should mention that in Figure 8 and Figure 10 the curve segments induced from $\partial D_{i}$ are only drawn schematically. In reality they are embedded on the boundary surface, but for simplicity, we draw them crossing each other but keep their endpoints fixed. This simplification will not affect our proofs because, later, when we make use of the Whitehead graphs, we only need information from the endpoints of the curve segments. Figure 9 and Figure 11 show us a genus 7 handlebody $\widetilde{H}$, which covers $H^{\prime}$. We take $\left\{X_{1}, X_{2}, X_{3}, Z_{1}, Z_{2}, Z_{3}, Y_{3}\right\}$ as a disk system for $\widetilde{H}$. Each $D_{i}$ is lifted to three disks $D_{i}^{j}, j=1,2,3$, whose boundaries are shown in Figure 9 and Figure 11 . Let $\widetilde{C}$ be the corresponding cover of $C$, then $\left\{D_{1}^{1}, D_{1}^{2}, D_{1}^{3}, D_{2}^{1}, D_{2}^{2}, D_{2}^{3}\right\}$ is a disk system for $\widetilde{C} . M_{K}^{3}$ has the induced Heegaard splitting $M_{K}^{3}=\widetilde{H} \cup_{\partial \widetilde{H}} \widetilde{C}$.

From the disk systems we see that the Heegaard splitting of $M_{K}^{3}$ is weakly reducible, since $\left\{D_{1}^{3}, D_{2}^{3}\right\}$ is disjoint form $\left\{X_{3}, Y_{3}, Z_{3}\right\}$. We also notice that the Heegaard splitting is actually stabilized and thus reducible. The longitude $\lambda$ is lifted to three copies, we show the one, $\widetilde{\lambda}$, disjoint from $X_{3}, Y_{3}$ and $Z_{3}$ in Figure 12 . Again for simplicity we did not draw it as embedded on the surface. We note that $\widetilde{\lambda}$ is disjoint from all $\partial D_{i}^{j}$,s.

Now we are going to show that the closed genus 2 surface $S$ obtained by compressing the Heegaard surface $\partial \widetilde{H}$ using the disks $D_{1}^{3}, D_{2}^{3}, X_{3}, Y_{3}$ and $Z_{3}$ is essential in $M_{K}^{3}$. Theorem 1.1 will then follow from Theorem 2.4.3 of [CGLS], namely the surface $S$ remains incompressible in every Dehn filling of $M_{K}^{3}$ with slope $m / n,(m, n)=1,|m|>1$, and since every such manifold is a cover of the manifold obtained by Dehn filling $M_{K}$ with slope $3 m / n,(3 m, n)=$ $1,|m|>1$.

It's enough to show that $S$ is incompressible in $M_{K}^{3}(2)$, which is the manifold obtained by Dehn filling $M_{K}^{3}$ with slope 2 . Let $C(6)$ be the genus 3 handlebody obtained by Dehn filling $C$ with slope $6 . M_{K}^{3}(2)$ has the induced Heegaard splitting $\widetilde{H} \cup \widetilde{C}(2)$, where $\widetilde{C}(2)$ is the genus 7 handlebody covering $C(6)$. Let $D$ be the meridian disk of the filling solid torus in $M_{K}$. By the definition of $D$, we can write out a presentation of $\partial D$ in terms of $x, y$ and 


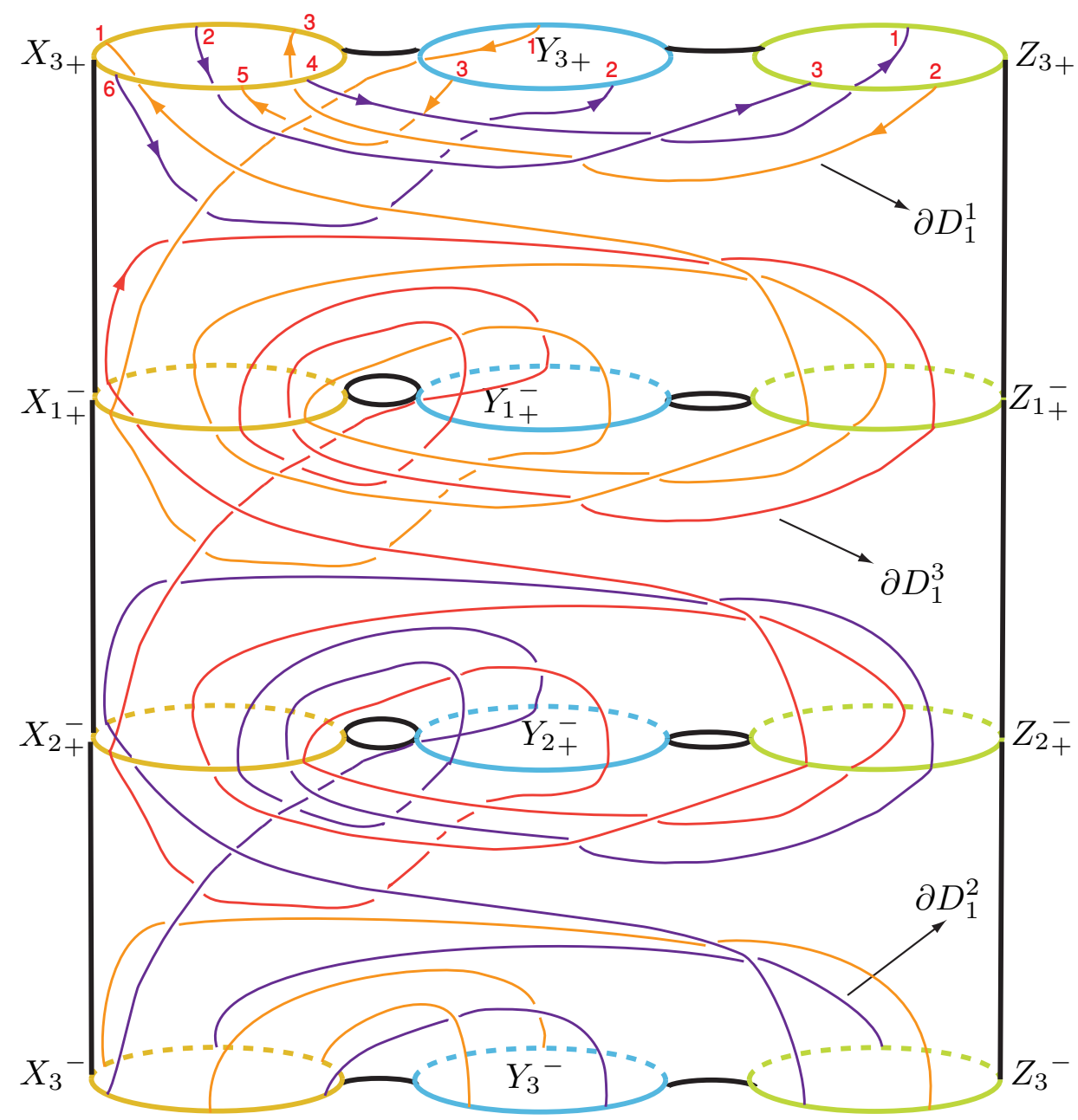

Figure 9: Lifts of $\partial D_{1}$ in the induced Heegaard surface of $M_{K}^{3}$.

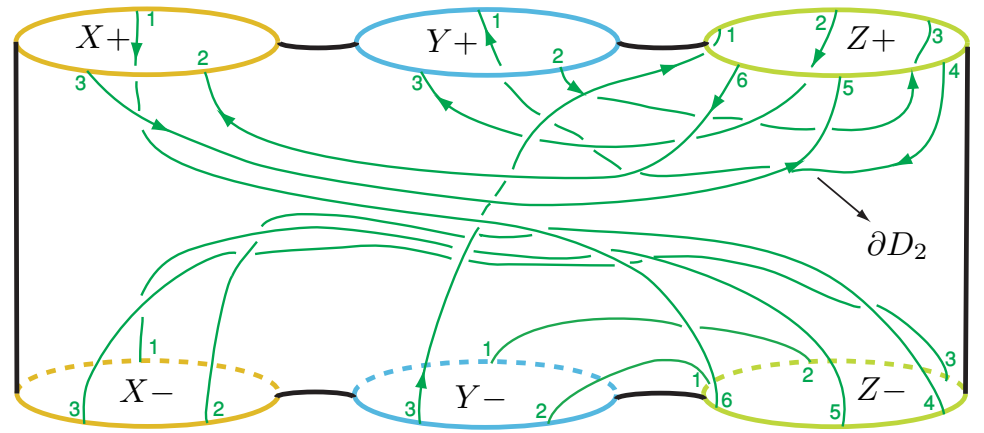

Figure 10: $\partial D_{2}$ in the resulting surface of cutting $\partial H^{\prime}$ open along $\{\partial X, \partial Y, \partial Z\}$. 


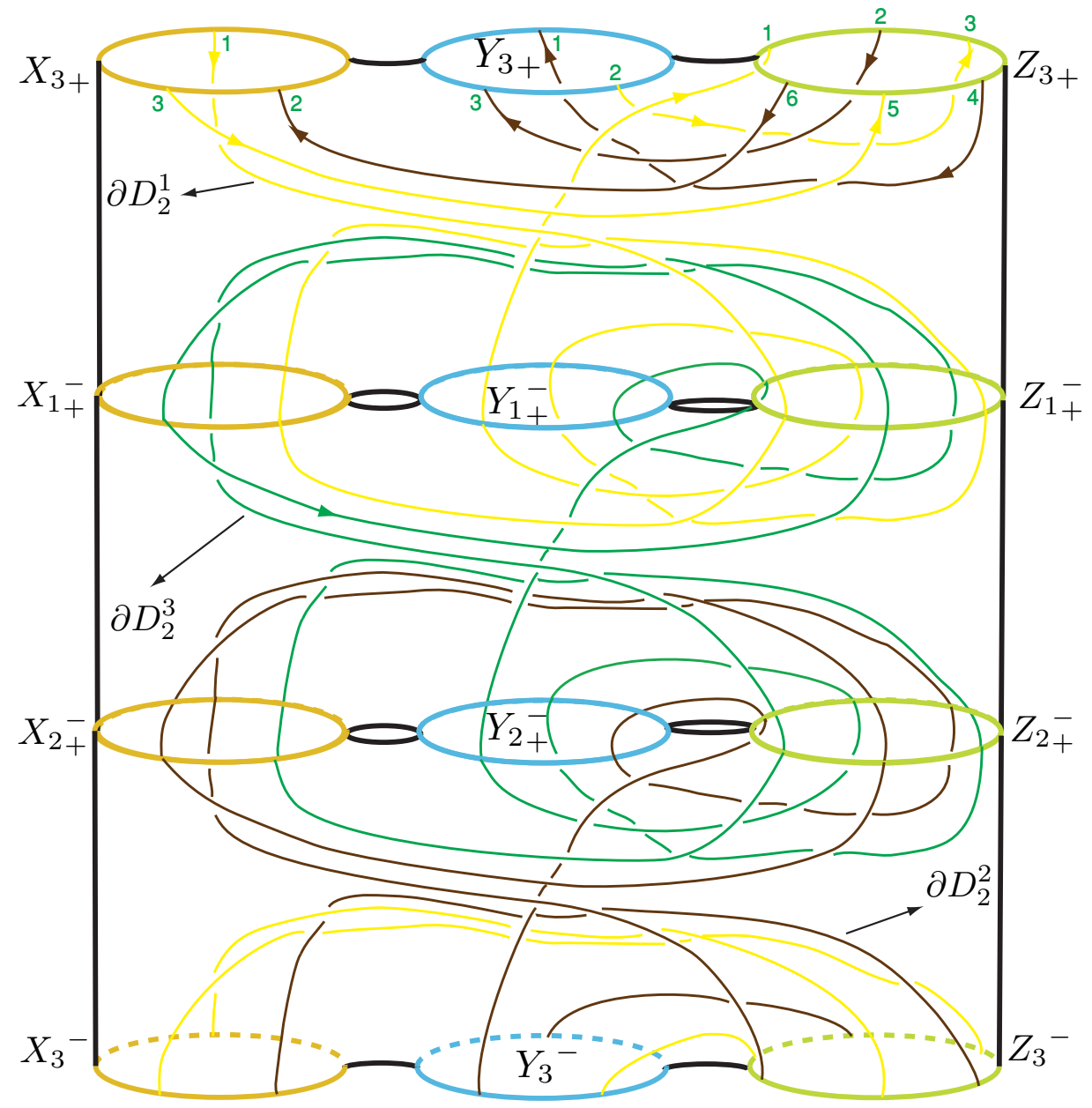

Figure 11: Lifts of $\partial D_{2}$ in the induced Heegaard surface of $M_{K}^{3}$. 


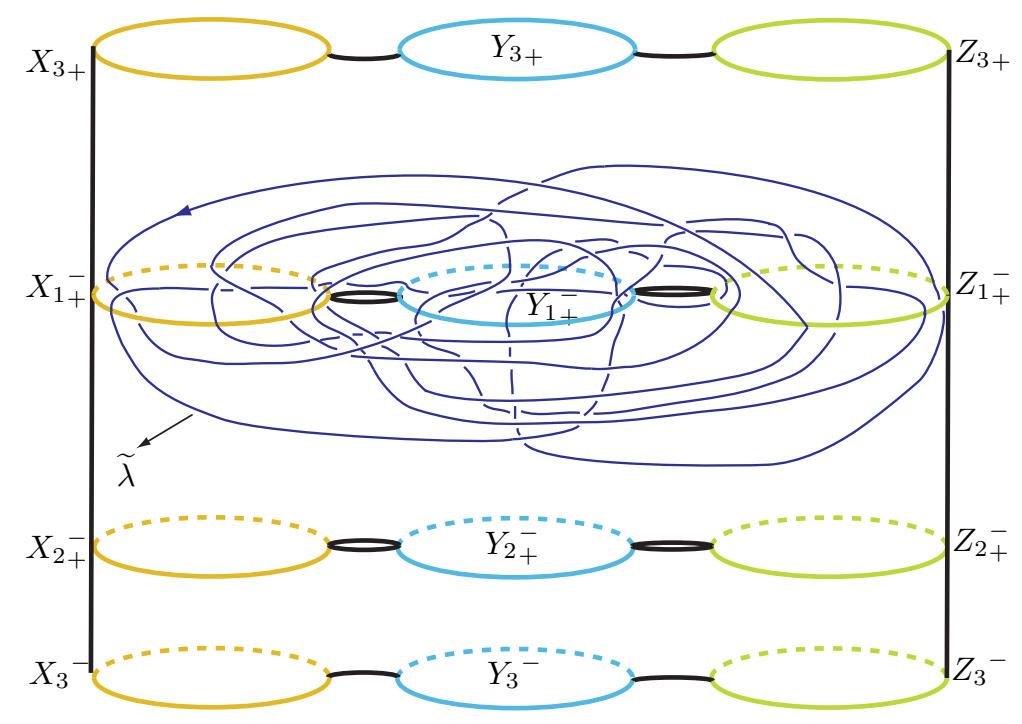

Figure 12: A lift of $\lambda$ in the induced Heegaard surface of $M_{K}^{3}$.

$z$ :

$$
\partial D=\lambda x^{6}=\left(y^{-1} x\right)\left(y^{-1} z\right)^{2}\left(x^{-1} z\right)\left(x^{-1} y\right)^{2}\left(z^{-1} y\right)\left(z^{-1} x\right)^{2} x^{6},
$$

A sketch of a lift of $\partial D$ in the induced Heeggard surface of $M_{K}^{3}, \partial \tilde{D}$, is shown in Figure 13 . Then $\left\{D_{1}^{1}, D_{1}^{2}, D_{1}^{3}, D_{2}^{1}, D_{2}^{2}, D_{2}^{3}, \tilde{D}\right\}$ is a disk system of $\tilde{C}(2)$.

Compressing $\widetilde{H}$ along $X_{3}, Y_{3}$ and $Z_{3}$, we get a handlebody $\bar{H}$ of genus 4 , with a disk system $\left\{X_{1}, X_{2}, Z_{1}, Z_{2}\right\}$. $\left\{\partial D_{1}^{3}, \partial D_{2}^{3}\right\}$ is a set of pairwise disjoint simple closed curves on $\partial \bar{H}$. By following $\partial D_{1}^{3}$ and $\partial D_{2}^{3}$ on Figure 9 and 11 , we can read off the Whitehead graph of $\left\{\partial D_{1}^{3}, \partial D_{2}^{3}\right\}$ with respect to $\left\{X_{1}, X_{2}, Z_{1}, Z_{2}\right\}$. The graph is shown in Figure 14 . The graph is connected and has no cut vertex. Thus $\partial \bar{H}-\left(\partial D_{1}^{3} \cup \partial D_{2}^{3}\right)$ is incompressible in $\bar{H}$. If we just look at the Whitehead graph of $\partial D_{1}^{3}\left(\partial D_{2}^{3}\right.$ respectively), i.e., the red part (green part respectively) of the graph, there are some valence one vertices in the graph. So by Corollary 2.2 both $\partial \bar{H}-\partial D_{1}^{3}$ and $\partial \bar{H}-\partial D_{2}^{3}$ are compressible. Hence by the multi-handle addition theorem (Theorem 2.3), the manifold $M_{1}=\bar{H} \cup\left(D_{1}^{3} \times I\right) \cup\left(D_{2}^{3} \times I\right)$ has incompressible boundary.

On the other hand, compressing the handlebody $\widetilde{C}(2)$ along $D_{1}^{3}$ and $D_{2}^{3}$, we get a handlebody $\bar{C}$ of genus 5 , with a disk system $\left\{D_{1}^{1}, D_{1}^{2}, D_{2}^{1}, D_{2}^{2}, \tilde{D}\right\} .\left\{\partial X_{3}, \partial Y_{3}, \partial Z_{3}\right\}$ is a set of pairwise disjoint simple closed curves on $\partial \bar{C}$.

To see the Whitehead graph of $\left\{\partial X_{3}, \partial Y_{3}, \partial Z_{3}\right\}$ with respect to $\left\{D_{1}^{1}, D_{1}^{2}, D_{2}^{1}, D_{2}^{2}, \tilde{D}\right\}$, we present a neighborhoods of $\partial X_{3}, \partial Y_{3}$ and $\partial Z_{3}$ in Figure 15. In the figure, we mark the positive and negative sides of each of $\left\{D_{1}^{1}, D_{1}^{2}, D_{2}^{1}, D_{2}^{2}, \tilde{D}\right\}$ by following the orientations and using right-hand rule, here we always let our thumbs point to the positive sides.

By following the oriented $\partial X_{3}, \partial Y_{3}$ and $\partial Z_{3}$, we can draw the Whitehead graph of 


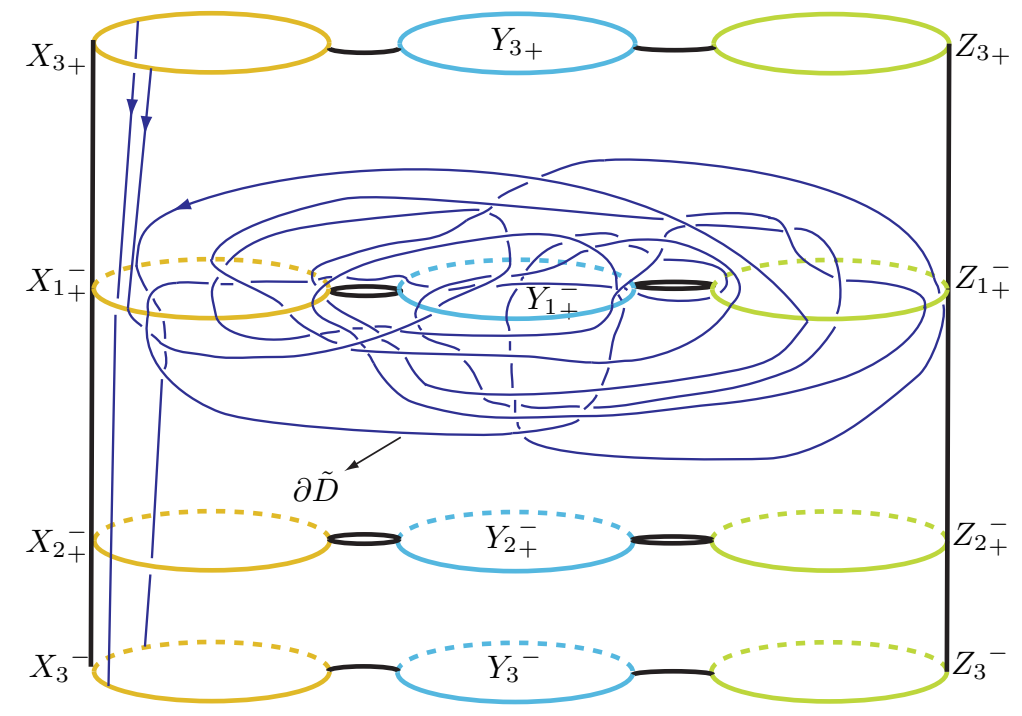

Figure 13: $\partial \tilde{D}$ in the derived Heegaard surface of $M_{K}^{3}$.

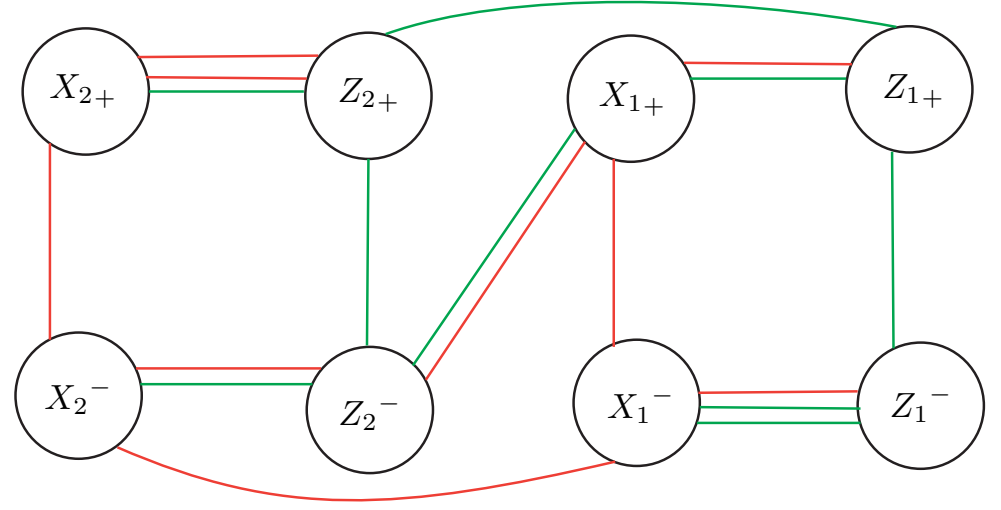

Figure 14: The Whitehead graph of $\left\{\partial D_{1}^{3}, \partial D_{2}^{3}\right\}$ with respect to $\left\{X_{1}, X_{2}, Z_{1}, Z_{2}\right\}$. 

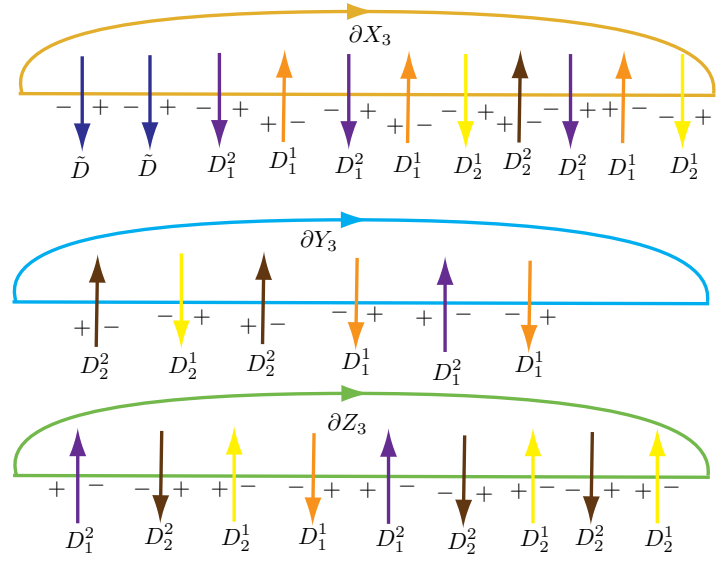

Figure 15: The neighborhoods of $\partial X_{3}, \partial Y_{3}$ and $\partial Z_{3}$.

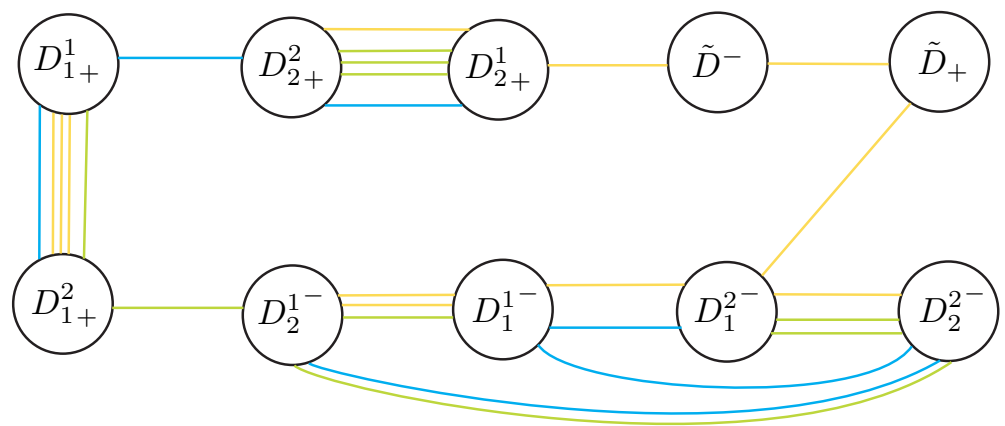

Figure 16: The Whitehead graph of $\left\{\partial X_{3}, \partial Y_{3}, \partial Z_{3}\right\}$ with respect to $\left\{D_{1}^{1}, D_{1}^{2}, D_{2}^{1}, D_{2}^{2}, \tilde{D}\right\}$.

$\left\{\partial X_{3}, \partial Y_{3}, \partial Z_{3}\right\}$ with respect to $\left\{D_{1}^{1}, D_{1}^{2}, D_{2}^{1}, D_{2}^{2}, \tilde{D}\right\}$, see Figure 16 . We can verify from this graph that the curve family $\left\{\partial X_{3}, \partial Y_{3}, \partial Z_{3}\right\}$ satisfies all the conditions of the multihandle addition theorem. In fact the graph is connected and has no cut vertex, so $\partial \bar{C}-$ $\left(\partial X_{3} \cup \partial Y_{3} \cup \partial Z_{3}\right)$ is incompressible in $\bar{C}$. If we just look at the graph of $\partial X_{3}, \partial Y_{3}$ or $\partial Z_{3}$, the graph is disconnected, so each of $\partial X_{3}, \partial Y_{3}$ and $\partial Z_{3}$ does not bind a free factor $F_{4}$ of $F_{5}$. The graph of $\partial Y_{3} \cup \partial Z_{3}$ is disjoint from $D$, so it does not bind $F_{5}$. The graph of $\partial X_{3} \cup \partial Y_{3}$ (respectively the graph of $\partial X_{3} \cup \partial Z_{3}$ ) has the form as two subgraphs connected by a path with only two vertices $\left\{\tilde{D}_{-}, \tilde{D}_{+}\right\}$. By Lemma 2.1. $\left\{\partial X_{3}, \partial Y_{3}\right\}$ (respectively $\left\{\partial X_{3}, \partial Z_{3}\right\}$ ) is separable in $\partial \bar{C}$, i.e. does not bind $F_{5}$. So all the conditions of the multi-handle addition theorem are satisfied. Thus the manifold $M_{2}=\bar{C} \cup\left(X_{3} \times I\right) \cup\left(Y_{3} \times I\right) \cup\left(Z_{3} \times I\right)$ has incompressible boundary.

Notice that $S=\partial M_{1}=\partial M_{2}$ up to isotopy, so $S$ is incompressible in $M_{K}^{3}(2)$. Also notice that $S$ is contained in $M_{K}^{3}$, so $S$ is an essential surface in $M_{K}^{3}$. This completes the proof of Theorem 1.1 for the case $K=(3,3,3)$. 
In general, for a pretzel knot $K=( \pm(2 i+1), \pm 3, \pm(2 j+1))$, the proof is similar. Up to knot equivalence and taking mirror images, we can divide our proof into 3 cases:

(1): $K=(2 i+1,3,2 j+1)$,

(2): $K=(-(2 i+1), 3,2 j+1)$,

$(3): K=(-(2 i+1), 3,-(2 j+1))$.

Let $H$ be a regular neighborhood of $K$ and the unknotting tunnels (which are chosen similarly as we did for the $(3,3,3)$-pretzel knot). After some proper deformation of $H$ in $S^{3}$, we can make the exterior $H^{\prime}$ of $H$ in $\mathbb{S}^{3}$ a standard handlebody of genus 3 . In the meantime we are still able to keep track of the boundaries of the meridians of the unknotting tunnels, $\partial D_{1}$ and $\partial D_{2}$, and get their final appearance in $\partial H^{\prime}$.

If we pick the disk system $\{X, Y, Z\}$ of $H^{\prime}$ and the generating set $\{x, y, x\}$ of $\pi_{1}\left(H^{\prime}\right)$ as before, we can read off a presentation of $\pi_{1}\left(M_{K}\right)$ from the curves $\partial D_{1}$ and $\partial D_{2}$ :

$$
\begin{gathered}
\pi_{1}\left(M_{K}\right)=\left\langle x, y, z: \partial D_{1}=1, \partial D_{2}=1\right\rangle, \\
\text { where } \partial D_{1}= \begin{cases}\left(x^{-1} y\right)^{i+1}\left(x y^{-1}\right)^{i}\left(x z^{-1}\right)^{2}\left(x^{-1} z\right) & \text { Case 1, } \\
\left(y^{-1} x\right)^{i}\left(y x^{-1}\right)^{i+1}\left(x z^{-1}\right)^{2}\left(x^{-1} z\right) & \text { Case 2, } \\
\left(y^{-1} x\right)^{i}\left(y x^{-1}\right)^{i+1}\left(x z^{-1}\right)^{2}\left(x^{-1} z\right) & \text { Case 3; }\end{cases} \\
\partial D_{2}= \begin{cases}\left(z y^{-1}\right)^{j+1}\left(z^{-1} y\right)^{j}\left(z^{-1} x\right)^{2}\left(z x^{-1}\right) & \text { Case 1, } \\
\left(z y^{-1}\right)^{j+1}\left(z^{-1} y\right)^{j}\left(z^{-1} x\right)^{2}\left(z x^{-1}\right) & \text { Case 2, } \\
\left(y z^{-1}\right)^{j}\left(y^{-1} z\right)^{j+1}\left(z^{-1} x\right)^{2}\left(z x^{-1}\right) & \text { Case 3. }\end{cases}
\end{gathered}
$$

By abelinization we get $H_{1}\left(M_{K}\right)=\mathbb{Z}=\langle y\rangle$, and $x=z=y$.

Similarly we can read off a word expression for an oriented longitude $\lambda$ as:

$$
\lambda= \begin{cases}\left(y^{-1} x\right)^{i}\left(y^{-1} z\right)^{j+1}\left(x^{-1} z\right)\left(x^{-1} y\right)^{i+1}\left(z^{-1} y\right)^{j}\left(z^{-1} x\right)^{2} & \text { Case 1, } \\ \left(y^{-1} x\right)^{i}\left(z^{-1} y\right)^{j}\left(z^{-1} x\right) z^{-1}\left(y x^{-1}\right)^{i}\left(z y^{-1}\right)^{j}\left(z x^{-1}\right) z & \text { Case 2, } \\ \left(x^{-1} z\right)\left(y^{-1} x\right)^{i}\left(y^{-1} z\right)^{j} y^{-1}\left(x z^{-1}\right)\left(y x^{-1}\right)^{i}\left(y z^{-1}\right)^{j} y & \text { Case 3. }\end{cases}
$$

Similarly, let $M_{K}^{3}$ be the 3-fold cyclic cover of $M_{K}$ corresponding to the homomorphism $\pi_{1}\left(M_{K}\right) \rightarrow H_{1}\left(M_{K}\right) \rightarrow \mathbb{Z}_{3}$ with the induced Heegaard splitting, which is also weakly reducible and stabilized because $\left\{X_{3}, Y_{3}, Z_{3}\right\}$ is disjoint from $\left\{\partial D_{1}^{3}, \partial D_{2}^{3}\right\}$. We can prove that the closed genus 2 surface $S$, obtained by compressing the Heegaard surface of $M_{K}^{3}$ using $X_{3}, Y_{3}, Z_{3}, D_{1}^{3}$ and $D_{2}^{3}$, is essential.

In fact, the Whitehead graph of $\left\{\partial D_{1}^{3}, \partial D_{2}^{3}\right\}$ with respect to $\left\{X_{1}, X_{2}, Z_{1}, Z_{2}\right\}$ is as shown in Figure 17, where parts (1)-(3) correspond to Case 1-Case 3 respectively. We can easily check that the graph satisfies all the conditions of the multi-handle addition theorem.

On the other hand, the Whitehead graph of $\left\{\partial X_{3}, \partial Y_{3}, \partial Z_{3}\right\}$ with respect to $\left\{D_{1}^{1}, D_{1}^{2}, D_{2}^{1}, D_{2}^{2}, \tilde{D}\right\}$ is shown in Figure 18, where $\tilde{D}$ is a meridian disk of the filling torus of the Dehn filling of 
(1)

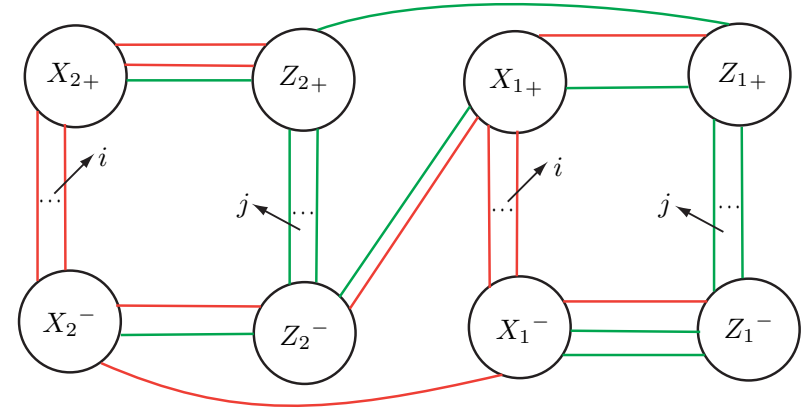

(2)

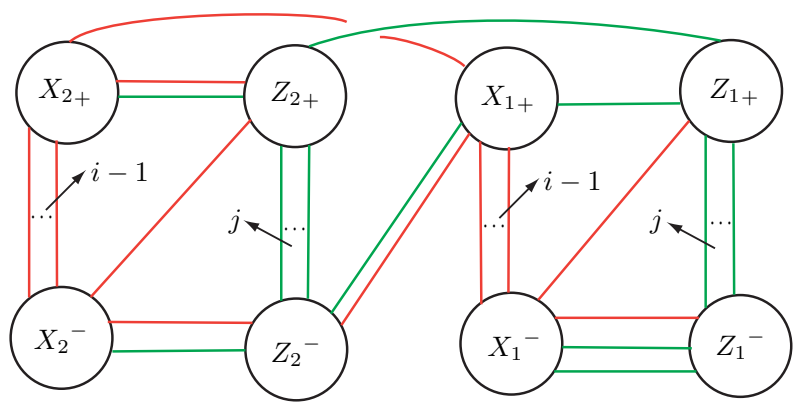

(3)

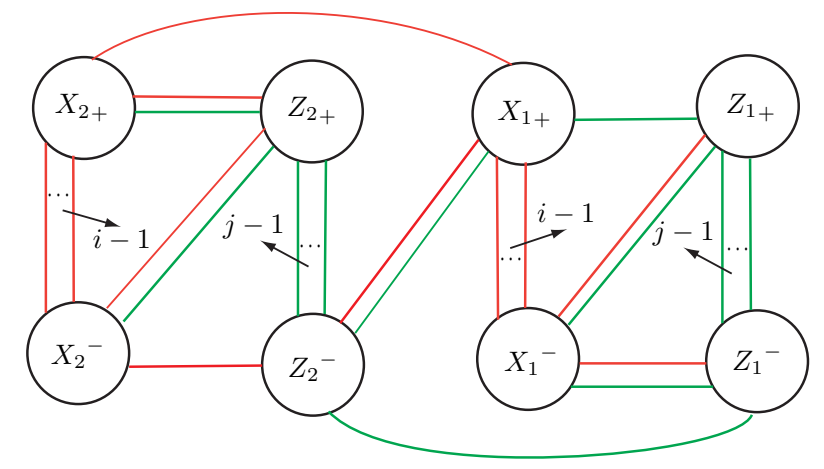

Figure 17: The Whitehead graph of $\left\{\partial D_{1}^{3}, \partial D_{2}^{3}\right\}$ with respect to $\left\{X_{1}, X_{2}, Z_{1}, Z_{2}\right\}$, (the general case). 
(1)

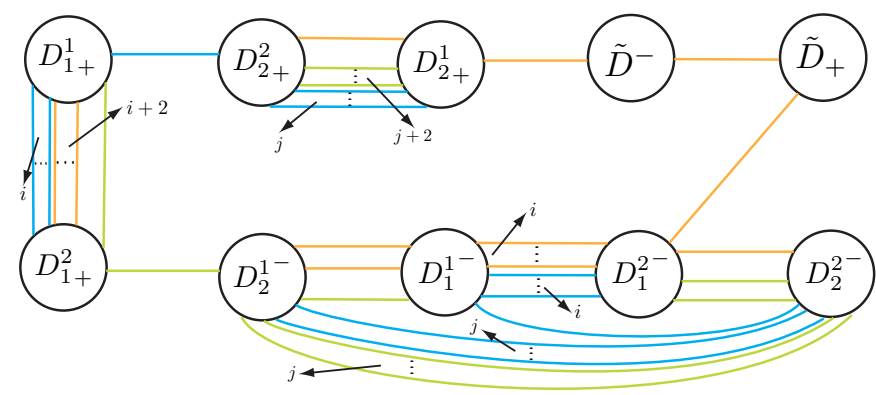

(2)

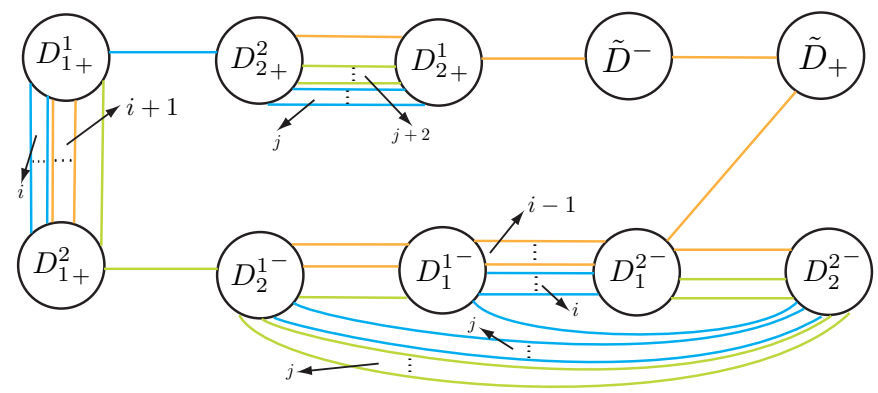

(3)

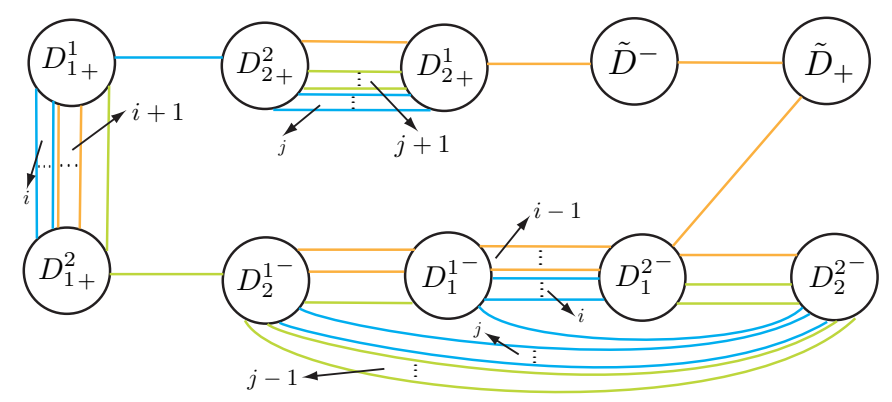

Figure 18: The Whitehead graph of $\left\{\partial X_{3}, \partial Y_{3}, \partial Z_{3}\right\}$ with respect to $\left\{D_{1}^{1}, D_{1}^{2}, D_{2}^{1}, D_{2}^{2}, \tilde{D}\right\}$, (the general case).

$M_{K}^{3}$ with slope 2 , and parts (1)-(3) correspond to Case 1-Case 3 respectively. We can also check that the graph satisfies all the conditions of the multi-handle addition theorem.

This finishes the proof of Theorem 1.1 .

Remark 3.1. All the lifted Heegaard splittings in Theorem 1.1 are stabilized (reducible). We can tell that from the Heegaard diagrams or from the Whitehead graphs.

\section{Another application of the method}

In this section, using the same method, we give a new proof of a part of a result of [O]. We show 


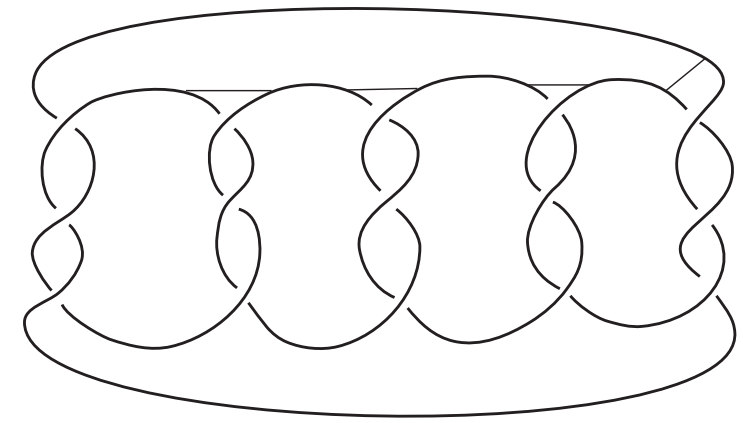

Figure 19: The $(3,3,3,3,3)$-pretzel knot with unknotting tunnels.

Proposition 4.1. If $K=\left(p_{1}, p_{2}, \cdots, p_{k}\right)$ is a pretzel knot with $k \geqslant 4, p_{i} \geqslant 3$, then $M_{K}=$ $S^{3} \backslash K$ contains a closed incompressible surface which remains incompressible in every closed 3-manifold obtained by a non-trivial Dehn filling on $M_{K}$.

Remark 4.2. Similar method can be used to prove the cases that $p_{i} \in \mathbb{Z}$ and $\left|p_{i}\right| \geqslant 3$.

According to Formula (1) in Section 2, a pretzel link $K=\left(p_{1}, \cdots, p_{k}\right)$ is a knot if and only if $k$ and all $p_{i}$ 's are odd or exactly one of the $p_{i}$ 's is even. So we may divide our proof into two cases:

Case 1: $k>4$ odd, all the $p_{i}$ 's are odd.

Case 2: One of the $p_{i}$ 's is even, $k \geqslant 4$.

Proof of Case 1: We will first prove this case for an example, $K=(3,3,3,3,3)$.

Figure 19 shows the $(3,3,3,3,3)$-pretzel knot $K$ with unknotting tunnels. A regular neighborhood, $H$, of the union of $K$ and the unknotting tunnels is a genus five handlebody. Let $D_{1}, \ldots, D_{4}$ be meridian disks of the four unknotting tunnels respectively and let $\lambda$ be a preferred longitude of $K$. We can deform $H$ in $\mathbb{S}^{3}$ such that its exterior $H^{\prime}$ is a standard handlebody in $\mathbb{S}^{3}$. At the same time we keep tracking the corresponding deformation of the curves $\partial D_{1}, \partial D_{2}, \partial D_{3}, \partial D_{4}$ and $\lambda$. Figure 20 shows the final position of these curves on the boundary surface of $H^{\prime}$. We pick a disk system $\left\{X_{1}, X_{2}, X_{3}, X_{4}, X\right\}$ of $H^{\prime}$ and a dual generating set $\left\{x_{1}, x_{2}, x_{3}, x_{4}, x\right\}$ of $\pi_{1}\left(H^{\prime}\right)$ as shown in Figure 20. We orient all the curves in $\partial H^{\prime}$ by the same method we used in the proof of Theorem 1.1. Figure 20 shows us a Heegaard splitting of the exterior $M_{K}$ of $K$, i.e., $M_{K}=H^{\prime} \cup C$, where $C$ is a compression body obtained by attaching four 1-handles to the positive boundary $\partial M_{K} \times[1]$ of $\partial M_{K} \times[0,1]$ with $\left\{D_{1}, D_{2}, D_{3}, D_{4}\right\}$ as a disk system.

This Heegaard splitting is weakly reducible, because $\{X\}$ is disjoint from $\left\{D_{2}, D_{3}\right\}$. We are now going to show that the genus two surface $S$ obtained by compressing the Heegaard surface $\partial H^{\prime}$ using $X, D_{2}$ and $D_{3}$ is incompressible in the manifold $M_{K}(m / n)$ which is a Dehn filling of $M_{K}$ with a nontrivial slope $m / n$. The closed manifold $M_{K}(m / n)$ has the 


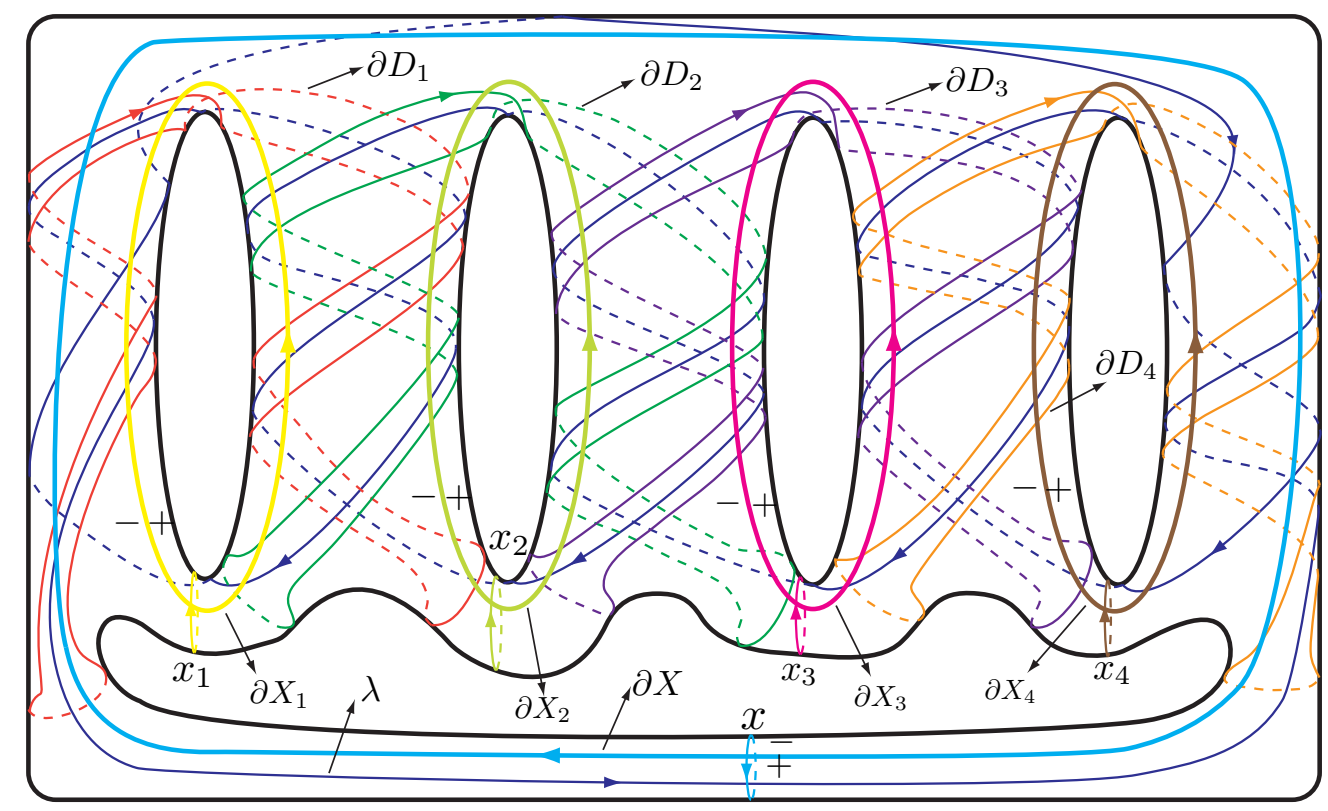

Figure 20: The deformation of $H, \lambda, \partial D_{1}, \partial D_{2}, \partial D_{3}, \partial D_{4}$.

induced Heegaard splitting $M_{K}(m / n)=H^{\prime} \cup_{\partial H^{\prime}} C(m / n)$, with $\left\{D_{1}, D_{2}, D_{3}, D_{4}, D(m / n)\right\}$ as a disk system of $C(\mathrm{~m} / n)$ (where $D(\mathrm{~m} / n)$ is a meridian disk of the Dehn filling torus of $C(m / n))$. Note that $\partial D(m / n)$ is a simple closed curve on $\partial M_{K}$, which can be drawn on a regular neighborhood of $x \cup \lambda$ as showed in Figure 21 .

Compressing $H^{\prime}$ along $X$, we get a handlebody $\bar{H}$ of genus four, with disk system $\left\{X_{1}, X_{2}, X_{3}, X_{4}\right\} .\left\{\partial D_{2}, \partial D_{3}\right\}$ is a set of simple closed curves on $\partial \bar{H}$. The Whitehead graph of $\left\{\partial D_{2}, \partial D_{3}\right\}$ with respect to $\left\{X_{1}, X_{2}, X_{3}, X_{4}\right\}$ is shown in Figure 22. The graph is connected and has two cut vertices $X_{3+}$ and $X_{2}{ }^{-}$. Applying the Whitehead automorphisms to these cut vertices (first $X_{3}^{+}$and then $X_{2}^{-}$), we get the graph shown in Figure 24 . This graph is connected and has no cut vertex. So $\partial \bar{H}-\left(\partial D_{2} \cup \partial D_{3}\right)$ is incompressible in $\bar{H}$. If we just look at the graph of $\partial D_{2}\left(\partial D_{3}\right.$ respectively) with respect to $\left\{X_{1}, X_{2}, X_{3}, X_{4}\right\}$, the graph is disconnected, which means $\partial \bar{H}-\partial D_{2}\left(\partial \bar{H}-\partial D_{3}\right.$ respectively) is compressible. So our graph satisfies all the conditions of the multi-handle addition theorem and thus the manifold $M_{1}=\bar{H} \cup\left(D_{2} \times I\right) \cup\left(D_{3} \times I\right)$ has incompressible boundary. Note that $\partial M_{1}=S$.

On the other hand, compress $C(\mathrm{~m} / n)$ along $D_{2}$ and $D_{3}$, we get a handlebody $\bar{C}$ of genus three with $\left\{D_{1}, D_{4}, D(m / n)\right\}$ as a disk system (for any $m / n \neq 1 / 0$ ). $\partial X$ is a simple closed curve on $\partial \bar{C}$. The Whitehead graph of $\partial X$ with respect of $\left\{D_{1}, D_{4}, D(m / n)\right\}$ is shown in Figure 25. The graph is connected and has no cut vertex. So $\partial \bar{C}-\partial X$ is incompressible in $\bar{C}$. Hence the manifold $M_{2}=\bar{C} \cup(X \times I)$ has incompressible boundary. Note that $\partial M_{2}=S$. 


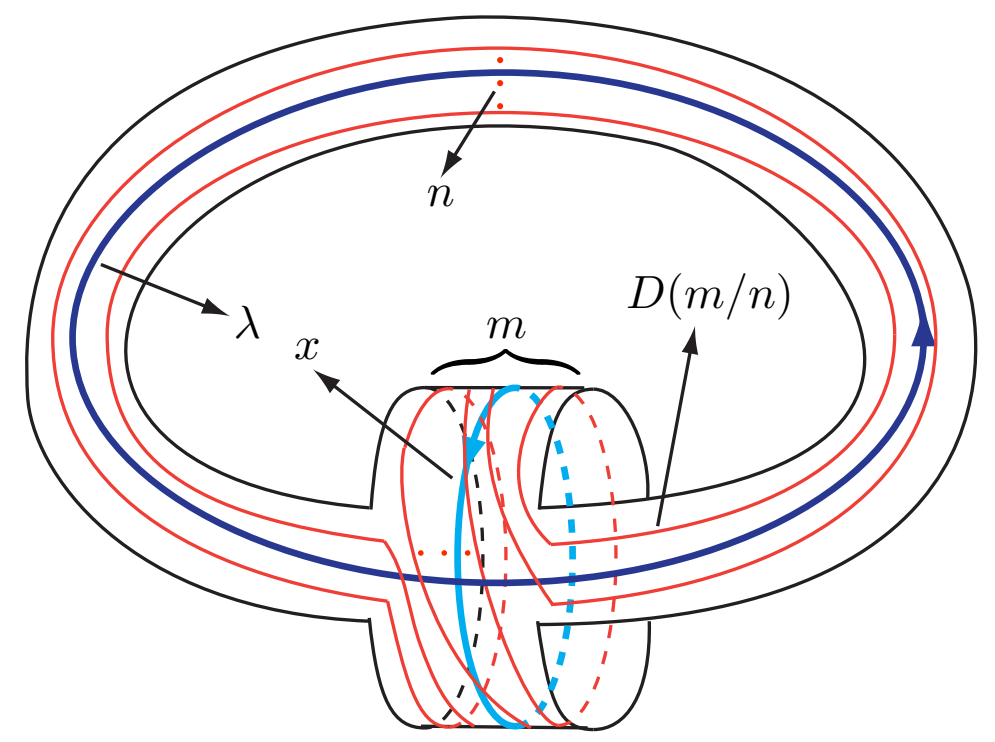

Figure 21: The filling slope of $C(m / n)$.

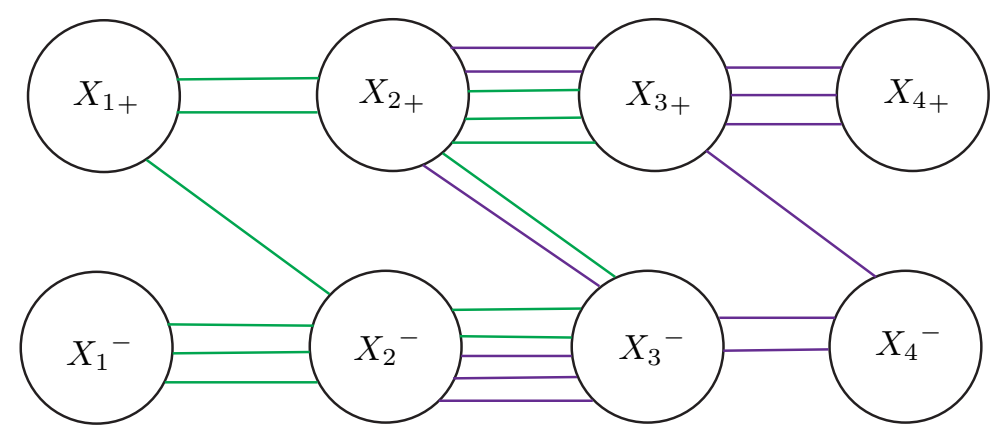

Figure 22: The Whitehead graph of $\left\{\partial D_{2}, \partial D_{3}\right\}$ with respect to $\left\{X_{1}, X_{2}, X_{3}, X_{4}\right\}$.

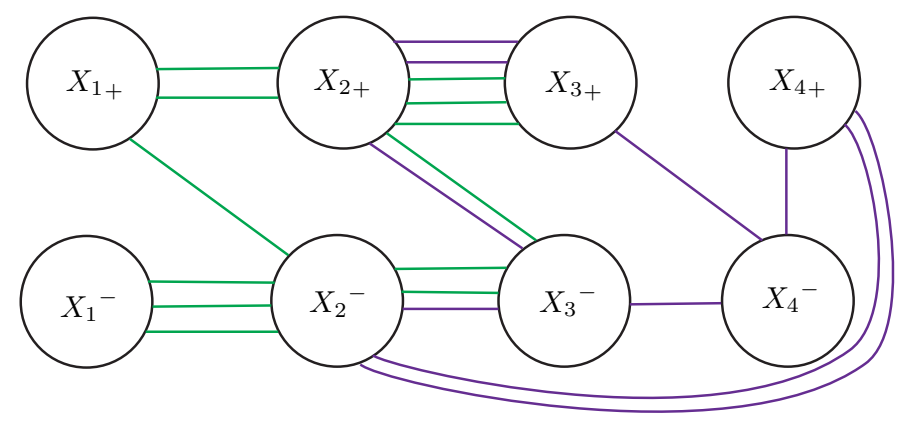

Figure 23: The transformed graph after applying the Whitehead algorithm to the graph in Figure 22 at its vertex $X_{3+}$. 


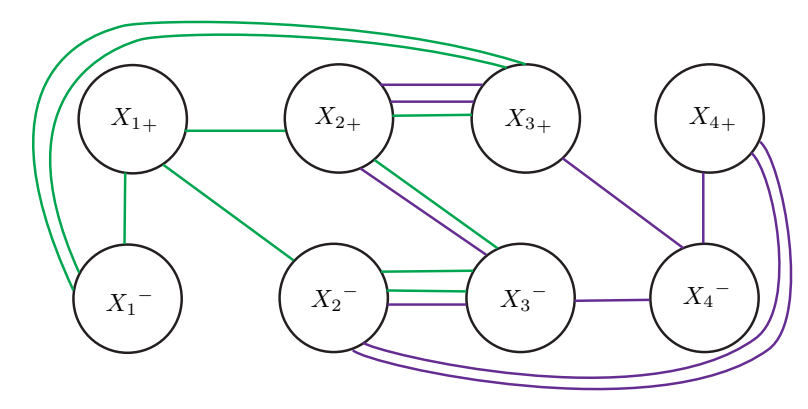

Figure 24: The transformed graph after applying the Whitehead algorithm to the graph in Figure 23 at its vertex $X_{2}^{-}$.

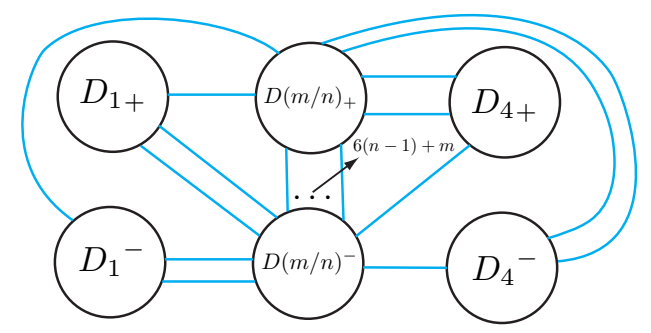

Figure 25: The Whitehead graph of $\partial X$ with respect to $\left\{D_{1}, D_{4}, D(m / n)\right\}$.

Now we have shown $S=\partial M_{1}=\partial M_{2}$ is incompressible in $M_{K}(m / n)$. Notice that $S$ is contained in $M_{K}$, so $S$ is also an essential surface in $M_{K}$. We finish the proof of Case 1 for the example $K=(3,3,3,3,3)$.

In general, for a pretzel knot $K=\left(p_{1}, p_{2}, \cdots, p_{k}\right)$ as in Case 1 , the proof is similar.

A regular neighborhood, $H$, of the union of $K$ and $k-1$ unknotting tunnels (similarly chosen as we did for the example) is a genus $k$ handlebody. We can deform $H$ so that its exterior $H^{\prime}$ is a standard handlebody in $\mathbb{S}^{3}$, and at the same time we may track the boundaries of the meridian disks of the unknotting tunnels, $\partial D_{1}, \cdots, \partial D_{k-1}$ and a preferred longitude $\lambda$. We pick a disk system of $H^{\prime}$ and a generating set of $\pi_{1}\left(H^{\prime}\right)$ in a similar way. The complement of $K, M_{K}$, has a Heegaard splitting, $M_{K}=H^{\prime} \cup C$, where $C$ is a compression body obtained by attaching $k-1$ 1-handles to the positive boundary $\partial M_{K} \times[1]$ of $\partial M_{K} \times[0,1] .\left\{X, X_{1}, \cdots, X_{k-1}\right\}$ is a disk system of $H^{\prime}$, and $\left\{D_{1}, \cdots, D_{k-1}\right\}$ is a disk system of $C$.

Since $\{X\}$ is disjoint from $\left\{D_{2}, \cdots, D_{k-2}\right\}$, this splitting is weakly reducible. We can show that the genus two surface $S$ obtained by compressing the Heegaard surface $\partial H^{\prime}$ using $X, D_{2}, \cdots, D_{k-2}$ is essential in the manifold $M_{K}(m / n)$ for every $m / n \neq 1 . M_{K}(m / n)$ has a Heegaard splitting $M_{K}(m / n)=H^{\prime} \cup_{\partial H^{\prime}} C(m / n) . C(m / n)$ is a genus $k$ handlebody with a meridian disk system $\left\{D_{1}, \cdots, D_{k-1}, D(m / n)\right\}$, where $D(m / n)$ is a meridian disk of the 


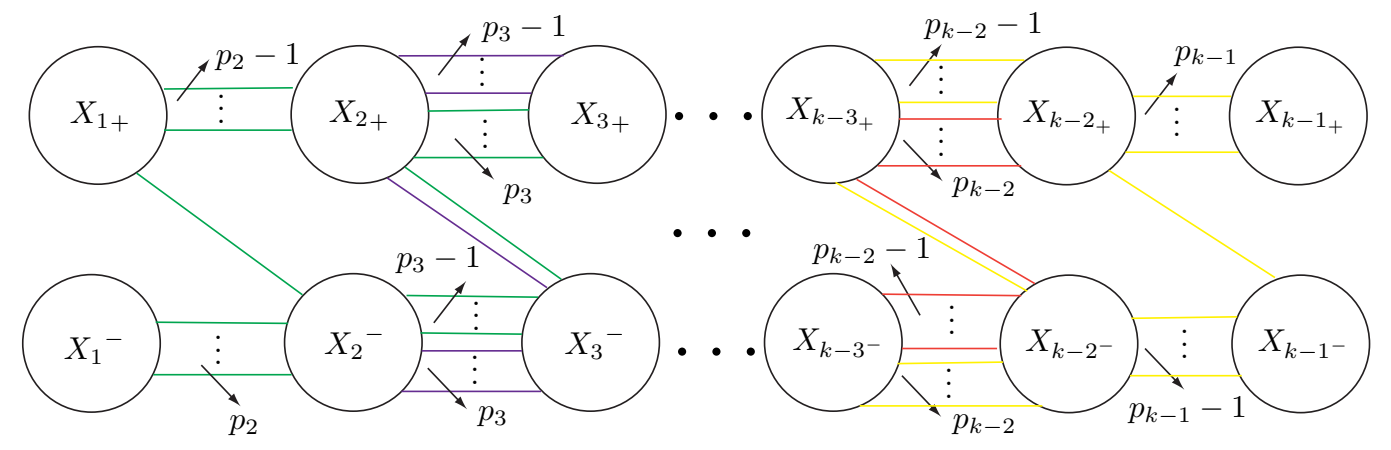

Figure 26: The Whitehead graph of $\left\{\partial D_{2}, \cdots, \partial D_{k-2}\right\}$ with respect to $\left\{X_{1}, \cdots, X_{k-1}\right\}$.

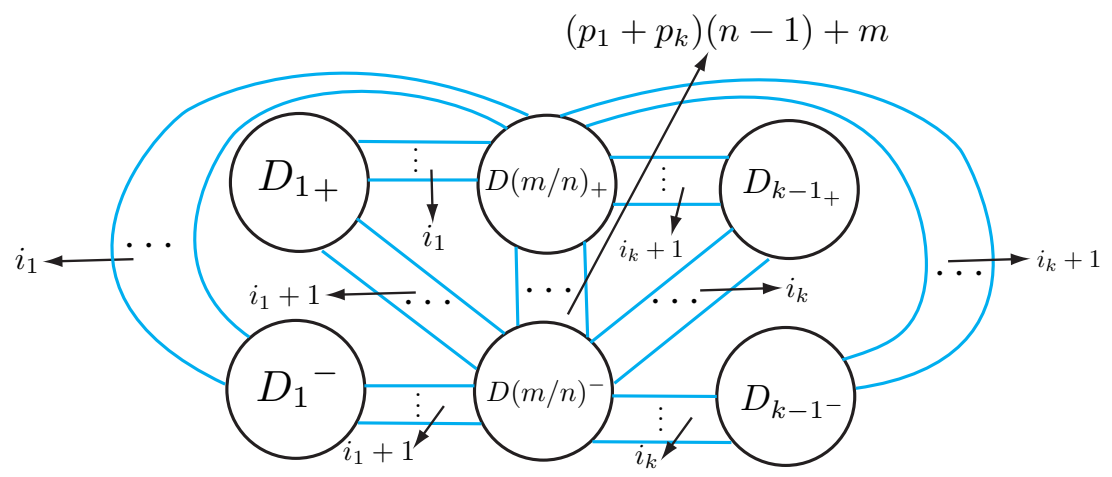

Figure 27: The Whitehead graph of $\partial X$ with respect $\left\{D_{1}, D_{k-1}, D(m / n)\right\}$.

Dehn filling torus of $C(m / n)$.

Compressing $H^{\prime}$ along $X$, we get a handlebody $\bar{H}$ of genus $k-1$, with disk system $\left\{X_{1}, \cdots, X_{k-1}\right\} \cdot\left\{\partial D_{2}, \cdots, \partial D_{k-2}\right\}$ is a set of simple closed curves on $\partial \bar{H}$. The Whitehead graph of $\left\{\partial D_{2}, \cdots, \partial D_{k-2}\right\}$ with respect to $\left\{X_{1}, \cdots, X_{k-1}\right\}$ is shown in Figure 26. We can check that this graph satisfies all the conditions of the multi-handle addition theorem (after applying some Whitehead automorphisms). So the manifold $M_{1}=\bar{H} \cup\left(D_{2} \times I\right) \cup \cdots \cup$ $\left(D_{k-2} \times I\right)$ has incompressible boundary $S$.

On the other hand, compress $C(m / n)$ along $D_{2}, \cdots, D_{k-2}$, we get a handlebody $\bar{C}$ of genus three with $\left\{D_{1}, D_{k-1}, D(m / n)\right\}$ as a disk system. $\partial X$ is a simple closed curve on $\partial \bar{C}$. The Whitehead graph of $\partial X$ with respect of $\left\{D_{1}, D_{k-1}, D(m / n)\right\}$ is shown in Figure 27. In the figure, $p_{1}=2 i_{1}+1$ and $p_{k}=2 i_{k}+1$. The graph is connected and has no cut vertex. So $\partial \bar{C}-\partial X$ is incompressible in $\bar{C}$. Thus the manifold $M_{2}=\bar{C} \cup(X \times I)$ has incompressible boundary.

So $S=\partial M_{1}=\partial M_{2}$ is incompressible in $M_{K}(m / n)$. Notice that $S$ is contained in $M_{K}$, 


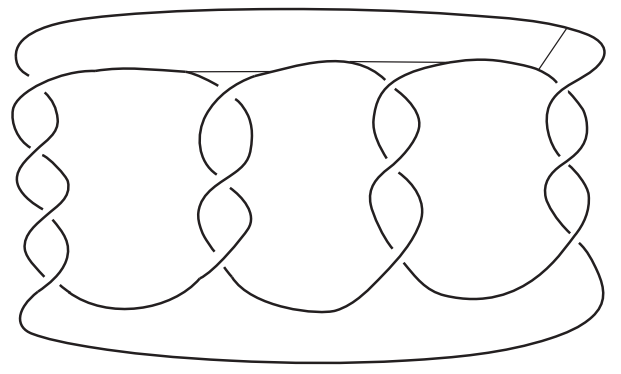

Figure 28: The (4,3,3,3)-pretzel knot with unknotting tunnels.

so $S$ is also an essential surface in $M_{K}$. We finish the proof of Case 1

Proof of Case 2: As before, we first prove this case for an example $K=(4,3,3,3)$. Note that, up to knot equivalence, we may assume that the left most tangle has even number of twists. Figure 28 shows us the (4,3,3,3)-pretzel knot with unknotting tunnels. A regular neighborhood, $H$, is a handle body of genus four.

As before, we deform $H$ so that its exterior looks like a standard handdlebody in $\mathbb{S}^{3}$. Figure 29 shows us the boundary surface of $H$, and $\partial D_{1}, \partial D_{2}, \partial D_{3}, \lambda$, after the deformation.

The knot complement $M_{K}=\mathbb{S}^{3} \backslash K$ has a Heegaard splitting $M_{K}=H^{\prime} \cup C$, where $H^{\prime}$ is the exterior of $H$ and $C$ is a compression body obtained by attaching three 1-handles to the positive boundary $\partial M_{K} \times[1] .\left\{X, X_{1}, X_{2}, X_{3}\right\}$ is a disk system of $H^{\prime}$, and $\left\{\partial D_{1}, \partial D_{2}, \partial D_{3}\right\}$ is a disk system of $C$.

This Heegaard splitting is weakly reducible, because $\{X\}$ is disjoint from $\left\{D_{2}\right\}$. By the similar argument, one can show that the genus two surface $S$ obtained by compressing $\partial H^{\prime}$ using $X$ and $D_{2}$ is essential in $M_{K}$. In fact $S$ remains incompressible in the manifold $M_{K}(m / n)$ for any $m / n \neq 1 / 0$.

Let $\bar{H}$ be the genus three handlebody obtained by compressing $H^{\prime}$ along $X$. It has a disk system $\left\{X_{1}, X_{2}, X_{3}\right\} . \partial D_{2}$ is a simple closed curve on $\partial \bar{H}$, and the Whitehead graph of $\partial D_{2}$ with respect to $\left\{X_{1}, X_{2}, X_{3}\right\}$ is shown in Figure 30 . This graph is connected and has two cut vertices $X_{2+}$ and $X_{2}{ }^{-}$. By applying the Whitehead algorithm to $X_{2+}$ we get a new graph as shown in Figure 31. The graph is connected and has no cut vertex. So $\partial \bar{H}-\partial D_{2}$ is incompressible in $\bar{H}$. Hence the manifold $M_{1}=\bar{H} \cup\left(D_{2} \times I\right)$ has incompressible boundary $S$.

On the other hand, compress $C(m / n)$ along $D_{2}$, we get a handlebody $\bar{C}$ of genus three with $\left\{D_{1}, D_{3}, D(m / n)\right\}$ as a disk system. $\partial X$ is a simple closed curve on $\partial \bar{C}$. The Whitehead graph of $\partial X$ with respect of $\left\{D_{1}, D_{3}, D(m / n)\right\}$ is shown in Figure 32, The graph is connected and has no cut vertex. So $\partial \bar{C}-\partial X$ is incompressible in $\bar{C}$. So the manifold $M_{2}=\bar{C} \cup(X \times I)$ has incompressible boundary. 


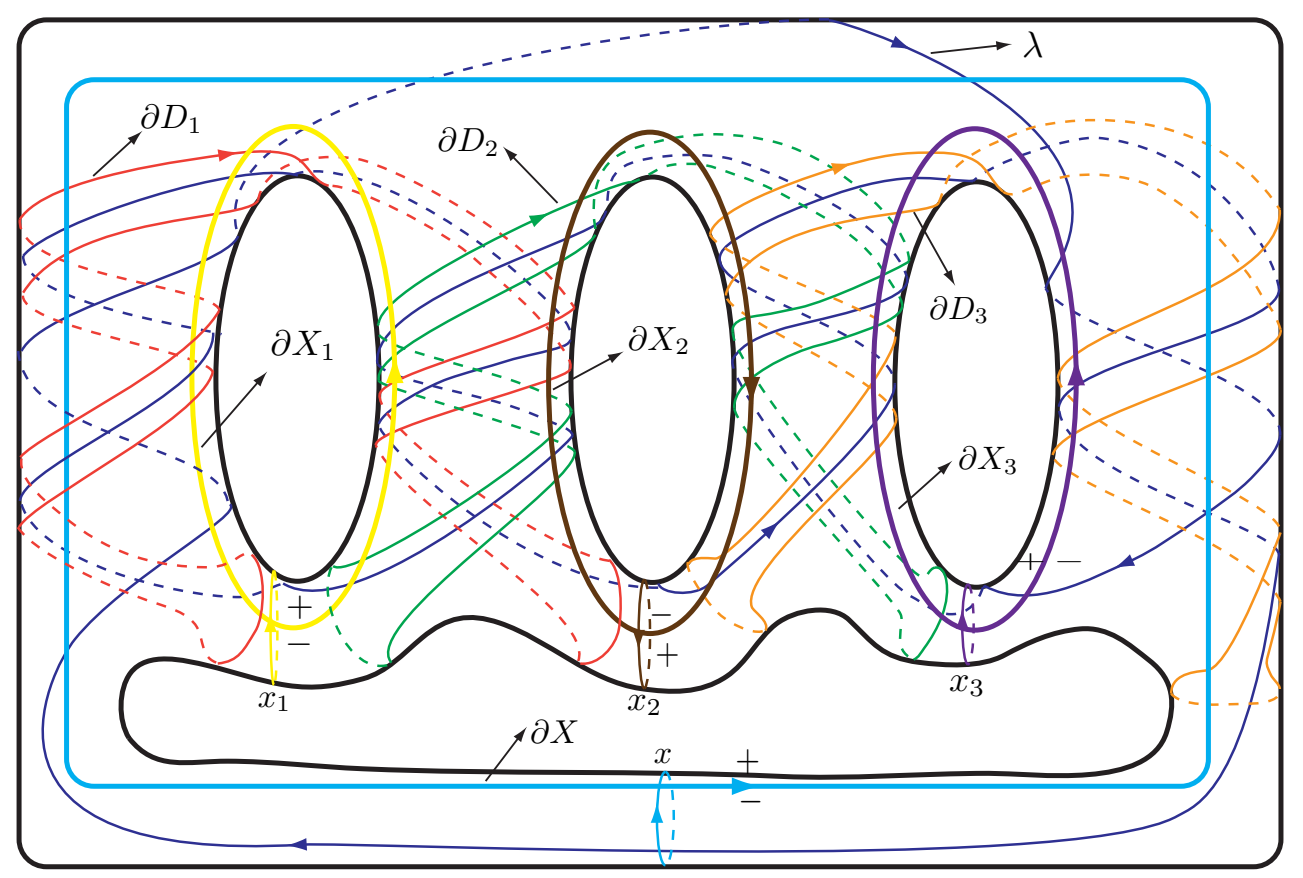

Figure 29: The deformation of $H, \lambda, \partial D_{1}, \partial D_{2}, \partial D_{3}$.

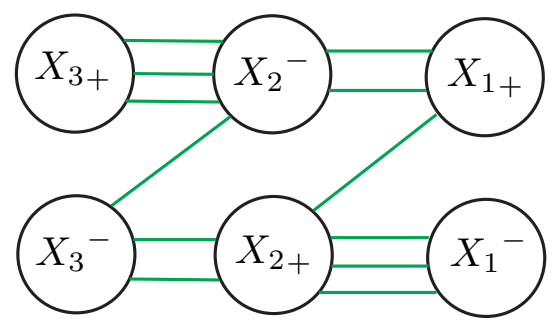

Figure 30: The Whitehead graph of $\partial D_{2}$ with respect to $\left\{X_{1}, X_{2}, X_{3}\right\}$.

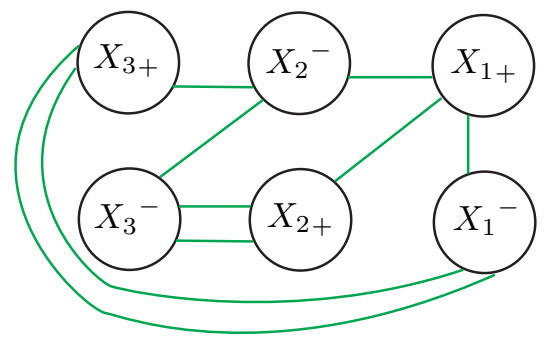

Figure 31: The resulting graph after applying the Whitehead automorphism to the graph in Figure 30 at its vertex $X_{2+}$. 


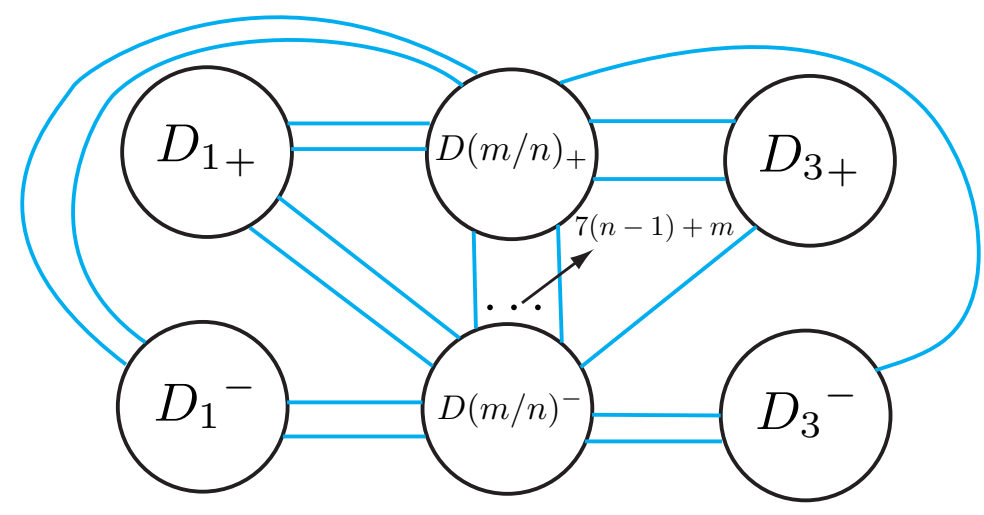

Figure 32: The Whitehead graph of $\partial X$ with respect to $\left\{D_{1}, D_{3}, D(m / n)\right.$.

Thus $S=\partial M_{1}=\partial M_{2}$ is incompressible in $M_{K}(m / n)=H^{\prime} \cup C(m / n)$. As $S$ is contained in $M_{K}$, it is also an essential surface in $M_{K}$.

In general, for a pretzel knot $K=\left(p_{1}, p_{2}, \cdots, p_{k}\right)$ as in Case 2 , the proof is similar.

A regular neighborhood, $H$, is a handle body of genus $k$. We deform $H$, such that it's exterior $H^{\prime}$ is a standard handlebody in $\mathbb{S}^{3}$. The knot complement $M_{K}=\mathbb{S}^{3} \backslash K$ has a Heegaard splitting $M_{K}=H^{\prime} \cup C$, where $C$ is a compression body obtained by attaching $k-1$ 1-handles to the positive boundary $\partial M_{K} \times[1] . \quad H^{\prime}$ has a meridian disk system $\left\{X, X_{1}, \cdots, X_{k-1}\right\}$, and $C$ has a meridian disk system $\left\{D_{1}, \cdots, D_{k-1}\right\}$.

Since $\{X\}$ is disjoint from $\left\{D_{2}, \cdots, D_{k-2}\right\}$, this Heegaard splitting is weakly reducible. By a similar argument, we can show that the genus two surface $S$ obtained by compressing $\partial H^{\prime}$ using $X$ and $D_{2}, \cdots, D_{k-2}$ is essential in $M_{K}$. Moreover, we can also show that $S$ remains incompressible in the manifold $M_{K}(m / n)$ for all $m / n \neq 1 / 0$. In fact if $\bar{H}$ is the genus $k-1$ handlebody obtained by compressing $H^{\prime}$ along $X$, it has a disk system $\left\{X_{1}, \cdots, X_{k-1}\right\} .\left\{\partial D_{2}, \cdots, \partial D_{k-2}\right\}$ is a family of simple closed curves on $\partial \bar{H}$, and the Whitehead graph of $\left\{\partial D_{2}, \cdots, \partial D_{k-2}\right\}$ with respect to $\left\{X_{1}, \cdots, X_{k-1}\right\}$ is shown in Figure 33. If $k$ is even, the graph looks like (1), if $k$ is odd, the graph looks like (2). We can check that this graph satisfies all the conditions of the multi-handle addition theorem. hence the manifold $M_{1}=\bar{H} \cup\left(D_{2} \times I\right) \cup \cdots \cup\left(D_{k-2} \times I\right)$ has incompressible boundary $S$.

On the other hand, let $C(m / n)$ be the handlebody obtained by Dehn filling $C$ with slope $m / n$ and let $D(m / n)$ be a meridian disk of the filling torus. Compress $C(m / n)$ along $D_{2}, \cdots, D_{k-2}$, we get a handlebody $\bar{C}$ of genus three, and $\left\{D_{1}, D_{k-1}, D(m / n)\right\}$ gives a disk system. $\partial X$ is a simple closed curve on $\partial \bar{C}$ whose Whitehead graph with respect of $\left\{D_{1}, D_{k-1}, D(m / n)\right\}$ is shown in Figure 34. The graph is connected and has no cut vertex. So $\partial \bar{C}-\partial X$ is incompressible in $\bar{C}$. Thus the manifold $M_{2}=\bar{C} \cup(X \times I)$ has incompressible boundary $S$. 


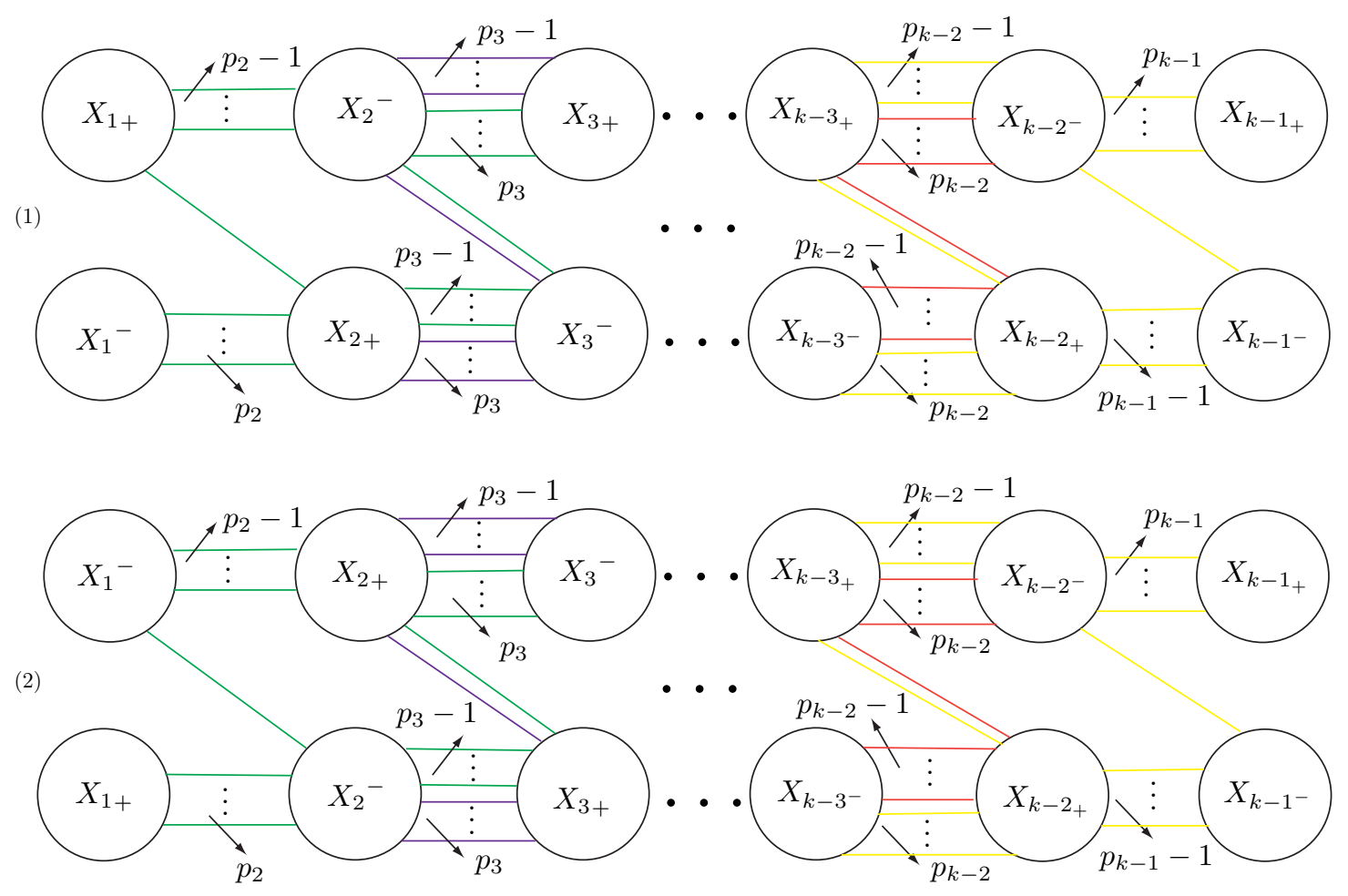

Figure 33: The Whitehead graph of $\left\{\partial D_{2}, \cdots, \partial D_{k-2}\right\}$ with respect to $\left\{X_{1}, \cdots, X_{k-1}\right\}$.

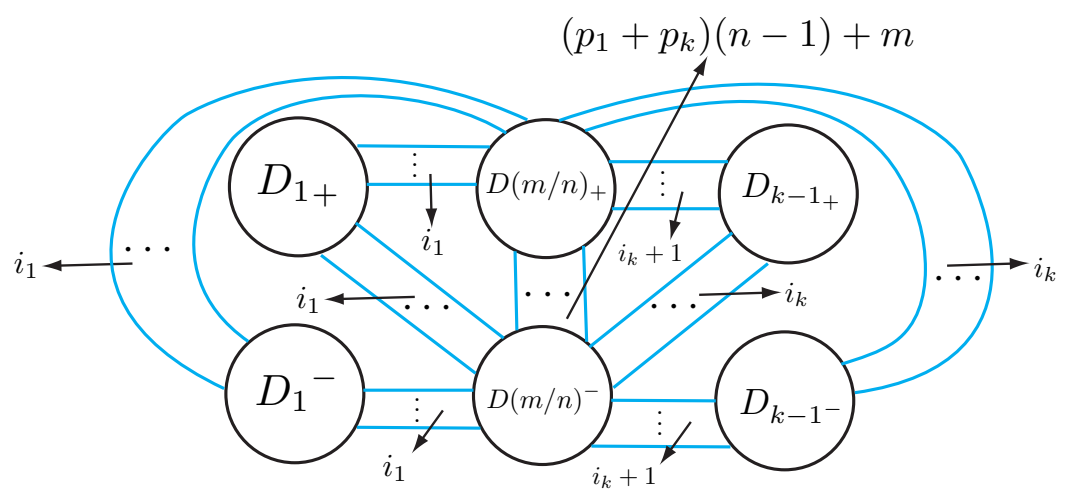

Figure 34: The Whitehead graph of $\partial X$ with respect to $\left\{D_{1}, D_{k-1}, D(m / n)\right.$. 
Now we have $S=\partial M_{1}=\partial M_{2}$, and so $S$ is incompressible in $M_{K}(m / n)=H^{\prime} \cup C(m / n)$. Notice that $S$ is contained in $M_{K}$, so $S$ is also an essential surface in $M_{K}$.

We finished the proof of Proposition 4.1 .

\section{References}

[CG] A. Casson and C. Gordon, Reducing Heegaard splittings, Topology Applications, 27 (1987) 275-283.

[CGLS] M. Culler, C. Gordon, J. Luecke and P. Shalen, Dehn surgery on knots, Ann. of Math., 125 (1978) 237-300, correction, Ann. of Math., 127 (1988) 663.

[H] A. Hatcher, On the boundary curves of incompressible surfaces, Pacific J. Math., 99 (1982), 373-377.

[La] M. Lackenby, Classification of alternating knots with tunnel number one, Comm. Anal. Geom. 13 (2005) no. 1, 151-185.

[Le] F. Lei, A proof of Przytycki's conjecture on n-relator 3-manifolds, Toplogy, 34 (1995), 473-476.

[MMZ] J. Masters, W. Menasco, and X. Zhang, Heegaard splittings and virtually Haken Dehn filling, New York Journal of Mathematics 10 (2004) 133-150.

[O] U. Oertel, Closed Incompressible Surfaces in Complements of Star Links, Pacific Journal of Mathematics, 111, No. 1 (1984) 209-230.

[RS] Y. Rieck and E. Sedgwick, Persistence of Heegaard structures under Dehn filling, Topology Appl. 109 (2001) 4153.

[S] J. Stallings, Whitehead graphs on handlebodies, Geometric group theory down under (Canberra, 1996), 317-330, de Gruyter, Berlin, 1999. 